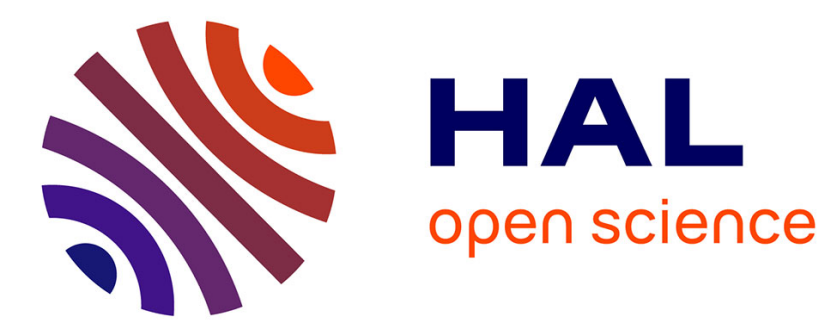

\title{
Ranking distributions of an ordinal variable
}

Nicolas Gravel, Brice Magdalou, Patrick Moyes

\section{To cite this version:}

Nicolas Gravel, Brice Magdalou, Patrick Moyes. Ranking distributions of an ordinal variable. Economic Theory, 2021, 71 (1), pp.33-80. 10.1007/s00199-019-01241-4 . hal-02383191

\section{HAL Id: hal-02383191 \\ https://hal.umontpellier.fr/hal-02383191}

Submitted on 10 Feb 2021

HAL is a multi-disciplinary open access archive for the deposit and dissemination of scientific research documents, whether they are published or not. The documents may come from teaching and research institutions in France or abroad, or from public or private research centers.
L'archive ouverte pluridisciplinaire $\mathbf{H A L}$, est destinée au dépôt et à la diffusion de documents scientifiques de niveau recherche, publiés ou non, émanant des établissements d'enseignement et de recherche français ou étrangers, des laboratoires publics ou privés. 


\title{
Ranking distributions of an ordinal variable
}

\author{
Nicolas Gravel ${ }^{1}$ (D) Brice Magdalou ${ }^{2} \cdot$ Patrick Moyes $^{3}$
}

\begin{abstract}
We establish an equivalence between three criteria for comparing distributions of an ordinal variable taking finitely many values. The first criterion is the possibility of going from one distribution to the other by a finite sequence of increments and/or Hammond transfers. The latter transfers are like the Pigou-Dalton ones, but with-out the requirement that the amount transferred be fixed. The second criterion is the unanimity of all comparisons of the distributions performed by a class of additively separable social evaluation functions. The third criterion is a new statistical test based on a weighted recursion of the cumulative distribution. We also identify an exact test for the possibility of going from one distribution to another by a finite sequence of Hammond transfers only. An illustration of the usefulness of our approach for evalu-ating distributions of self-reported happiness level is also provided.
\end{abstract}

Keywords Inequality $\cdot$ Ordinal $\cdot$ Transfers $\cdot$ Dominance $\cdot$ Distributions

JEL Classification D3 · D63

\section{Introduction}

When can we say that one distribution of a cardinally meaningful variable among a group of agents is more equal than another? One of the greatest achievements of the modern theory of inequality measurement is the demonstration, made by Hardy et al.

\footnotetext{
This article is a significant revision of a paper entitled "ranking distributions of an ordinal attribute" released as AMSE working paper no. 2014-50. In preparing this revision, we benefited from detailed comments made by Ramses Abdul Naga on the earlier paper. We also received valuable suggestions from Salvador Barbera, Yves Sprumont and Alain Trannoy. Any remaining defects in this work are of course our own. We also gratefully acknowledge financial support from the French Agence Nationale de la Recherche (ANR) through three contracts: Measurement of Ordinal and Multidimensional Inequalities (ANR-16-CE41-0005), Preference for Redistribution (ANR-15-CE26-0004) and Challenging Inequalities: A Indo-European perspective (ANR-18-EQUI-0003).
}

$\triangle$ Nicolas Gravel

Nicolas.gravel@csh-delhi.com

Extended author information available on the last page of the article 
(1952) and popularized among economists by Kolm (1969), Atkinson (1970), Dasgupta et al. (1973), Sen (1973) and Fields and Fei (1978) that the following three answers to this question are equivalent:

1. When one distribution has been obtained from the other by a finite sequence of mean-preserving transfers from a poorer to a richer agent, namely Pigou-Dalton transfers.

2. When one distribution would be considered better than the other by all utilitarian planners who assume that agents convert income into utility by the same concave function.

3. When the Lorenz curve associated with one distribution lies nowhere below, and at least somewhere above, that of the other.

This equivalence result ties together three a priori distinct aspects of inequality measurement. The first is an elementary transformation of the distribution that captures, in a crisp fashion, the nature of the equalization at stake. The second aspect is the ethical principle underlying utilitarianism or, more generally, additively separable social evaluation. The third aspect is the empirically implementable criterion underlying Lorenz dominance.

The current paper addresses the very same question in the case where the distributed variable is measured in an ordinal fashion. The last twenty years or so have indeed witnessed an extensive use of data involving distributions of variables such as access to basic services, educational achievements, health outcomes or self-declared happiness - to mention just a few-whose units of measurement are not cardinally meaningful. When comparing distributions of such variables, most researchers simply ignore their ordinal nature and treat them, just like income, as magnitudes that can be 'summed,' or 'transferred' across agents. Examples include Castelló-Clement and Doménech $(2002,2008)$ (discussing inequality indices on human capital) and Pradhan et al. (2003) (decomposing Theil indices applied to the heights of children under 36 month interpreted as a measure of health). The last 15 years, however, have seen the emergence of a literature [see, for example, Allison and Foster (2004), Abul-Naga and Yalcin (2008), Apouey (2007), Zheng (2008, 2011), Kobus and Milós (2012), Kobus (2015), Chakravarty and Maharaj (2015), Cowell and Flachaire (2017), Bosmans et al. (2018) and Fleurbaey and Maniquet (2019)] that takes due account of the ordinal nature of the available information on the attributes that affect individuals well-being when appraising the distributions of those attributes that form a normative standpoint.

A difficulty raised by the normative evaluation of a distribution of an ordinal variable is that of defining an adequate notion of inequality reduction. What does it mean for an ordinal variable to be 'more equally distributed' than another ? It is no use invoking the notion of Pigou-Dalton transfer for answering that question. A Pigou-Dalton transfer is, in effect, the operation by which an agent transfers a given quantity of the distributed variable to another agent. This notion of 'given quantity' is obviously meaningless when applied to a variable that is only ordinally measured.

Some forty years ago, Hammond (1976) proposed, in the context of social choice theory, a minimal equity principle that is explicitly concerned with distributions involving an ordinally measurable variable. According to Hammond's principle, a change in the distribution that reduces the gap between two agents endowed with different 
values of the variable is inequality reducing irrespective of whether or not the gain from the poor recipient is equal to the loss from the rich giver. The purely ordinal nature of Hammond transfers qualifies them, in our view, as highly plausible instances of clear inequality reduction.

The main contribution of this paper is to identify a normative dominance criterion and a statistically implementable criterion that are each equivalent to the notion of equalization underlying Hammond transfer. It does so in the specific but empirically important case where the ordinal variable can take only a finite number of different values corresponding to as many categories. Such variables are sometimes referred to as ordered categorical. Our choice of the ordered categorical variable case has specific implications for the Hammond equity principle. Indeed, as is well known in social choice theory [see, for example, D'Aspremont and Gevers (1977), D’Aspremont (1985), Hammond (1979) and Sen (1977)], when the ordinal variable is allowed to vary continuously, the Hammond equity principle is closely related to the lexicographic extension of the Maximin—or Leximin—ordering. Hammond (1979) has even shown that the Leximin ordering is the only anonymous, Pareto-inclusive and transitive ranking of all vectors in $\mathbb{R}^{n}$ that is strictly sensitive to Hammond transfers. ${ }^{1}$ As shown in this paper, this tight connection between the Leximin ordering and Hammond transfers becomes significantly looser when attention is restricted to distributions of an ordered categorical variable.

Concerning the class of normative principles in the spirit of answer 2. above, we stick to the tradition of comparing distributions by means of an additively separable social evaluation function. Each category of the variable is thus assigned a numerical value by some function, and distributions are compared on the basis of the sum, taken over all agents, of these values. While this normative approach can be considered utilitarian (if the value assigned to the attribute is interpreted as 'utility'), it does not need to be. One could also interpret the function more generally as an 'advantage function' reflecting the value assigned to each category by some ethical observer. If the variable is considered to be good for the agent, the advantage function can be assumed to be increasing with respect to the categories. We show in this paper that, in order for a ranking of distributions based on an additively separable social evaluation function to be sensitive to Hammond transfers, it is necessary and sufficient for the advantage function to satisfy a somewhat strong concavity property. Specifically, any increase in category obtained from some initial level must increase the advantage more than any increase obtained at some higher category, no matter what the latter increase is. Because of this result, we consider the ranking of distributions provided by the unanimity of all additively separable rankings based on an advantage function that is strongly concave in this sense.

The first empirically implementable criterion that we consider is, to the best of our knowledge, a new one. It can be viewed as the ordinal counterpart of the generalized Lorenz criterion (Kolm 1969; Shorrocks 1983) widely used for comparing distributions of a cardinal variable. Its construction is based on a curve that we call the $H$-curve, by reference to the Hammond principle of transfers to which it is closely related. The $H$-curve, easy to construct, is defined as follows. It starts by assigning to

\footnotetext{
1 A nice discussion of this result is provided by Tungodden (2000).
} 
the lowest category the fraction of the population that belongs to it. It then proceeds recursively, for any higher category, by adding together the relative frequency of the population belonging to this category and twice the value assigned by the curve to the immediately preceding category. The criterion that we propose, and that we call $H$-dominance, is for the dominating distribution to have an $H$-curve nowhere above and somewhere below that of the dominated one. We demonstrate that having a distribution that $H$-dominates another is equivalent to the possibility of going from the latter to the former by a finite sequence of Hammond transfers and/or increments in the variable. We also show that $H$-dominance coincides with the unanimity of all additively separable aggregations of advantage functions that are increasing and strongly concave in the manner described above.

$H$-dominance combines equity and efficiency considerations through, respectively, Hammond transfers and increments. Hence, it does not identify 'inequality reduction' only. The standard way to capture the notion of 'inequality reduction' in the classical cardinal setting is by applying the generalized Lorenz criterion to two distributions with the same mean. It is indeed well known that the Lorenz domination of one distribution over another is equivalent, when the two distributions have the same mean, to the possibility of going from the dominated to the dominating distribution by a finite sequence of Pigou-Dalton transfers. There is obviously no meaningful analogue to the 'mean' in an ordinal setting. Nevertheless, we know from the theory of majorization [see Marshall et al. (2011)] that Lorenz dominance between two distributions of a cardinal variable with the same mean is no more than the intersection of two independent majorization quasi-orderings that do not assume anything about the mean: weak supermajorization (the generalized Lorenz criterion) and weak submajorization (a criterion, dual to the previous one).

In this paper, we parallel the route taken in Marshall et al. (2011) by exploring a criterion, dual to $H$-dominance, which is based on what we call the $\bar{H}$-curve of a distribution. This curve is constructed just like the $H$-curve, except that it starts from 'above' rather than from 'below,' and iteratively cumulates the survival function rather than the cumulative distribution function. We then establish that $\bar{H}$-dominance coincides with the possibility of going from the dominated to the dominating distribution by a finite sequence of either Hammond transfers and/or decrements. Just as with the weak submajorization and weak supermajorization criteria in the cardinal setting, we demonstrate that the ranking of ordinal distributions generated by the intersection of the dominance criteria $H$ and $\bar{H}$ coincides with the possibility of going from the dominated to the dominating distribution by a finite sequence of Hammond transfers only.

Another result established in this paper concerns the behavior of the dominance criteria $H$ and $\bar{H}$ when the finite grid used to define the categories is 'refined.' We show that the Leximin criterion (resp. anti-Leximax) can be viewed as the limit of $H$-dominance (resp. $\bar{H}$ dominance) when the number of different categories of the variable becomes large. It follows that the intersection of the anti-Leximax and the Leximin criteria is the limit of the intersection of $H$-and $\bar{H}$-dominance criteria when the number of categories becomes large. This result echoes the analysis of Gravel et al. (2019) in a continuous setting which shows that having a vector in $\mathbb{R}^{n}$ that dominates another by both the Leximin and the anti-Leximax criteria is equivalent 
to the possibility of going from the dominated to the dominating vector by a finite sequence of Hammond transfers.

The plan of the rest of the paper is as follows. The next section introduces the notation and presents the elementary transformations, the normative criteria and the implementation criteria we consider when the attribute can take finitely many different values. The main results identifying the elementary transformations underlying the dominance criteria $H$ and $\bar{H}$ are stated and proved in the third section. The fourth section compares the discrete setting and the classical social choice setting originally used for the Hammond equity principle and examines the behavior of the implementation criteria when the number of categories is enlarged. The fifth section illustrates the usefulness of the criteria for comparing distributions of self-reported life satisfaction across countries based on the Gallup World Poll for 2014. The sixth section concludes.

\section{Three perspectives for comparing distributions of an ordinal variable}

\subsection{Main notation}

We consider distributions of an ordinal variable among a fixed number, say $n$, of agents. ${ }^{2}$ We assume that there are $k$ (with $k \geq 3$ ) different values that the variable can take which can be interpreted as categories, ordered from the worst to the best. We let $\mathcal{C}=\{1, \ldots, k\}$ denote the set of categories. The fact that the variable is ordinal means that the integers $1, \ldots, k$ assigned to the different categories have no significance other than reflecting the ordering of the categories (from worst to best). Hence, any comparative statement made on two distributions in which the variable is measured by the list of numbers $1, \ldots, k$ would be unaffected if this list was replaced by the list $f(1), \ldots, f(k)$, generated by any strictly increasing real-valued function $f$. We adopt throughout an anonymous perspective according to which 'the identity of the agents' does not matter. This enables us to describe any distribution or society $s$ as a particular list $\left(n_{1}^{s}, \ldots, n_{k}^{s}\right)$ of $k$ nonnegative integers satisfying $\sum_{h=1}^{k} n_{h}^{s}=n$, where $n_{h}^{s}$ denotes the number of agents in society $s$ who are in category $h$.

\subsection{Elementary transformations}

The definition of these transformations lies at the very heart of the problem of comparing alternative distributions of the variable of interest. These transformations are intended to capture in a crisp and concise fashion intuitions about the meaning of 'equalizing' or 'gaining in efficiency' (among others). In defining the transformations in the present context, it is important to ensure that they are consistent with the ordinal nature of the variable. In this paper, we discuss three such transformations.

\footnotetext{
2 As is standard in distributional analysis since at least Dalton (1920), distributions involving a varying number of agents can be compared by means of the principle of population replication. (Replicating a distribution any number of times is a matter of social indifference).
} 
The first, called increment, is hardly new. It captures the idea—somewhat related to efficiency - that moving an agent from a category to a better one is a good thing, ceteris paribus. We actually formulate this principle in the following minimalist fashion.

Definition 1 (Increment) We say that society $s$ has been obtained from society $s^{\prime}$ by means of an increment, if there exist $j \in\{1, \ldots, k-1\}$ such that:

$$
\begin{aligned}
& n_{h}^{s}=n_{h}^{s^{\prime}}, \forall h \neq j, j+1 ; \\
& n_{j}^{s}=n_{j}^{s^{\prime}}-1 ; n_{j+1}^{s}=n_{j+1}^{s^{\prime}}+1 .
\end{aligned}
$$

In words, society $s$ has been obtained from society $s^{\prime}$ by an increment if the move from $s^{\prime}$ to $s$ is the sole result of the move of one agent from a category $j$ to the immediately superior category $(j+1)$. The second transformation, called decrement, can simply be viewed as the reverse operation.

Definition 2 (Decrement) We say that society $s$ has been obtained from society $s^{\prime}$ by means of a decrement if and only if society $s^{\prime}$ has been obtained from $s$ by an increment in the sense of Definition 1 .

The third elementary transformation, hereafter referred as Hammond transfer, is the one underlying the equity principle put forward by Hammond (1976) some forty years ago. This principle considers that a reduction in the quantity of the variable assigned to one agent that is compensated by an increase in the quantity of the variable assigned to another agent is a good thing if the loser is, after and before the reduction, better off than the winner. The formal definition of such a transfer is as follows.

Definition 3 (Hammond transfer) We say that society $s$ is obtained from society $s^{\prime}$ by means of a Hammond' transfer, if there exist categories $1 \leq g<i \leq j<l \leq k$ such that:

$$
\begin{aligned}
& n_{h}^{s}=n_{h}^{s^{\prime}}, \forall h \neq g, i, j, l ; \\
& n_{g}^{s}=n_{g}^{s^{\prime}}-1 ; n_{i}^{s}=n_{i}^{s^{\prime}}+1 ; \\
& n_{j}^{s}=n_{j}^{s^{\prime}}+1 ; n_{l}^{s}=n_{l}^{s^{\prime}}-1 .
\end{aligned}
$$

While a reduction in an agent's endowment that is compensated by an increase in that of another agent may be viewed as the result of a 'transfer', it should be noted that, unlike standard Pigou-Dalton transfers, the increment in the agent initially in category $g$ is not necessarily equal to the decrement in the agent initially in category $l$. Since comparing the gains and losses of an ordinal attribute is meaningless, the Hammond transfer can be viewed as the natural analogue, in the ordinal setting, of the Pigou-Dalton transfer. ${ }^{3}$

\footnotetext{
3 Observe that a Pigou-Dalton transfer is nothing else than a Hammond transfer for which the indices $g, i$, $j$ and $l$ of Definition 3 satisfy the additional condition that $(i-g)=(l-j)$. See Fishburn and Lavalle (1995) or Chakravarty and Zoli (2012) for analysis of Pigou-Dalton transfers in a discrete setting and Abul-Naga (2018) for an examination, in a similar setting, of the closely related notion of Lorenz maximality.
} 


\subsection{Normative evaluation}

We assume that alternative societies are compared by some ethical observer who uses an additively separable criterion. Such an ethical observer would consider that society $s$ is normatively better than society $s^{\prime}$ if:

$$
\sum_{h=1}^{k} n_{h}^{s} \alpha_{h} \geq \sum_{h=1}^{k} n_{h}^{s^{\prime}} \alpha_{h}
$$

holds for some list of numbers $\left(\alpha_{1}, \ldots, \alpha_{k}\right) \in \mathcal{A} \subseteq \mathbb{R}^{k}$, which can be interpreted as numerical valuations of the corresponding categories. These valuations may reflect subjective utility (if a utilitarian perspective is adopted), but a non-welfarist interpretation is also possible, which can be axiomatically justified. [See, for example, Gravel et al. (2011).] We emphasize that the normative valuations of the categories that appear in Inequality (6) must be distinguished from the numbers assigned to the categories in the empirical data from which the categories are constructed. The latter numbers are meaningless. They only serve as indexing the categories from the worst to the best. The normative valuations $\left(\alpha_{1}, \ldots, \alpha_{k}\right)$ that appear in Inequality (6) are not meaningless. They reflect the normative values assigned to the categories by the ethical observer. As we will see, these normative valuations will need to satisfy rather specific properties in order to generate ethical evaluation that are consistent with Hammond transfers.

Some care must, however, be taken in avoiding that the normative evaluation exercise underlying Inequality (6) is unduly sensitive to particular choices of numbers $\alpha_{j}$. The typical way of achieving this in dominance analysis is to require Inequality (6) to hold over a wide class of such lists $\left(\alpha_{1}, \ldots, \alpha_{k}\right)$ of $k$ numbers.

\subsection{Implementation criteria}

Three implementation criteria are considered in this paper. The first one, called first-order (stochastic) dominance, is standard. It compares the values taken by the cumulative distribution function $F(\cdot ; s)$ associated with every society $s$ and defined, for every category $i \in \mathcal{C}$, by:

$$
F(i ; s)=\sum_{h=1}^{i} n_{h}^{s} / n .
$$

with the convention-if necessary-that $F(0 ; s)=0$. A society $s$ would then be considered to dominate society $s^{\prime}$ at the first order if the inequality $F(i ; s) \leq F\left(i ; s^{\prime}\right)$ is observed for every category $i \in\{1, \ldots, k-1\}$.

Hereafter, we will also refer to the survival function $\bar{F}(\cdot ; s)$ associated with a society $s$, which is defined by $\bar{F}(\cdot ; s)=1-F(\cdot, s)$ or, equivalently, by $\bar{F}(k ; s)=0$ and by:

$$
\bar{F}(i ; s)=\sum_{h=i+1}^{k} n_{h}^{s} / n
$$


for every $i=0, \ldots, k-1$. Hence, $\bar{F}(i ; s)$ is the fraction of the population in $s$ that is in a strictly better category than $i$.

The second implementation criterion, called $H$-dominance, is based on the following $H$-curve, defined for any society $s$ and any $i \in \mathcal{C}$, by:

$$
H(i ; s)=\sum_{h=1}^{i}\left(2^{i-h}\right) n_{h}^{s} / n
$$

We say that society $s H$-dominates society $s^{\prime}$ if and only if the inequality $H(i ; s) \leq$ $H\left(i ; s^{\prime}\right)$ holds for every category $i \in\{1, \ldots, k-1\}$. A few remarks can be made about the $H$-curve. First, it verifies:

$$
H(1 ; s)=F(1 ; s)=n_{1}^{s} / n
$$

and:

$$
H(i ; s)=\sum_{h=1}^{i-1}\left(2^{i-h-1}\right) F(h ; s)+F(i ; s), \forall i \in\{2, \ldots, k\} .
$$

Observe that Expression (11) makes it clear that first-order dominance implies $H$ dominance. We also note that the different values of $H(\cdot ; s)$ are nested. For any $i \in$ $\{2, \ldots, k\}$, we have:

$$
H(i ; s)=2 H(i-1 ; s)+F(i ; s)-F(i-1 ; s)=2 H(i-1 ; s)+n_{i}^{s} / n .
$$

Hence, by successive decomposition, we obtain, for any $i \in\{2, \ldots, k\}$ :

$$
H(i ; s)=\left(2^{j}\right) H(i-j ; s)+\sum_{h=0}^{j-1}\left(2^{h}\right) n_{i-h}^{s} / n, \forall j \in\{1, \ldots, i-1\} .
$$

In plain English, $H(i ; s)$ is a (specifically) weighted sum of the fractions of the population in $s$ that are in weakly worse categories than $i$. The weight assigned to the fraction of the population in category $h$ (for $h \leq i$ ) in that sum is $2^{i-h}$. Hence, the weights are (somewhat strongly) decreasing with respect to the categories. A nice feature of the $H$-curve is its recursive construction, described in Expressions (10) and (12), which is quite similar to that underlying the cumulative distribution curve. Indeed, for any $i \in\{2, \ldots, k\}$, the cumulative distribution $F(\cdot ; s)$ can be recursively written as $F(i ; s)=F(i-1 ; s)+n_{i}^{s} / n$.

The third implementation criterion examined herein, called $\bar{H}$-dominance, is dual to $H$-dominance. For any society $s$, the $\bar{H}$-curve is defined by $\bar{H}(k ; s)=0$ and:

$$
\bar{H}(i ; s)=\sum_{h=i+1}^{k}\left(2^{h-i-1}\right) n_{h}^{s} / n, \forall i \in\{1, \ldots, k-1\} .
$$


We say that society $s \bar{H}$-dominates society $s^{\prime}$ if and only if the inequality $\bar{H}(i ; s) \leq$ $\bar{H}\left(i ; s^{\prime}\right)$ holds for every category $i \in\{1, \ldots, k-1\}$. We note that the $\bar{H}$-curve is constructed under exactly the same recursive principle as the $H$-curve, but starting with the highest category, and iterating with the survival function rather than with the standard cumulative distribution function. Indeed, we have:

$$
\bar{H}(k-1 ; s)=\bar{F}(k-1 ; s)=n_{k}^{s} / n
$$

and also:

$$
\bar{H}(i ; s)=\sum_{h=i+1}^{k-1}\left(2^{h-i-1}\right) \bar{F}(h ; s)+\bar{F}(i ; s), \forall i \in\{1, \ldots, k-2\} .
$$

Moreover the different values of $\bar{H}(\cdot ; s)$ are nested so that, for any $i \in\{1, \ldots, k-2\}$, we have:

$$
\bar{H}(i ; s)=2 \bar{H}(i+1 ; s)+\bar{F}(i ; s)-\bar{F}(i+1 ; s)=2 \bar{H}(i+1 ; s)+n_{i+1}^{s} / n .
$$

Expressions (15) and (17) for $\bar{H}(\cdot ; s)$ echo the recursive construction of $\bar{F}(\cdot ; s)$, recalling that $\bar{F}(i ; s)=\bar{F}(i+1 ; s)+n_{i+1}^{s} / n$. Finally, just as in Expression (13), we obtain, for any $i \in\{1, \ldots, k-2\}$ :

$$
\bar{H}(i ; s)=\left(2^{j}\right) \bar{H}(i+j ; s)+\sum_{h=1}^{j}\left(2^{h-1}\right) n_{i+h}^{s} / n, \forall j \in\{1, \ldots, k-i-1\} .
$$

As illustrated in Sect. 5, the $H$ and $\bar{H}$ curves are easy to use and draw. As will also be seen in the next section, the two dominance criteria that they generate serve as perfect diagnostic test of the possibility of moving from the dominated to the dominating distribution by Hammond transfers and increments for $H$-dominance, or Hammond transfers and decrements for $\bar{H}$-dominance. Moreover, the additional criterion provided by the intersection of $\bar{H}$ - and $H$-dominance happens to provide an exact test of the possibility of going from the dominating to the dominated distribution by a finite sequence Hammond transfers only.

We end this section by pointing out the links between some of these notions of dominance. Specifically, we show that first-order dominance of a society $s^{\prime}$ by a society $s$ entails the $H$-dominance of society $s^{\prime}$ by $s$ and the $\bar{H}$-dominance of society $s$ by $s^{\prime}$. All the proofs are relegated in 'Appendix.'

Proposition 1 Suppose $s$ and $s^{\prime}$ are two societies such that $F(i ; s) \leq F\left(i ; s^{\prime}\right)$ for all categories $i \in\{1, \ldots, k-1\}$. Then, $H(i ; s) \leq H\left(i ; s^{\prime}\right)$ and $\bar{H}\left(i ; s^{\prime}\right) \leq \bar{H}(i ; s)$ for all $i \in\{1, \ldots, k-1\}$. 


\section{Equivalence results}

This section establishes a few theorems connecting, on the one hand, normative comparison of two societies as per Condition (6) over specific classes of collections of normative valuations $\left(\alpha_{1}, \ldots, \alpha_{k}\right) \in \mathbb{R}^{k}$ and, on the other hand, specific implementable criteria as well as the possibility of going from the dominated to the dominating distribution by appropriate elementary transformations.

We start with the notions of increment and decrement. Suppose that we are comparing two societies on the basis of Inequality (6) for some list $\left(\alpha_{1}, \ldots, \alpha_{k}\right)$ of normative valuations. What properties must these normative valuations satisfy for such a comparison to always consider an increment (decrement) as a social improvement? It should come as no surprise that the answer to this question is that the $k$ valuations must belong to the following sets:

$$
\mathcal{A}_{F}=\left\{\left(\alpha_{1}, \ldots, \alpha_{k}\right) \in \mathbb{R}^{k} \mid \alpha_{1} \leq \cdots \leq \alpha_{k}\right\} \text { (for increments) }
$$

and

$$
\mathcal{A}_{\bar{F}}=\left\{\left(\alpha_{1}, \ldots, \alpha_{k}\right) \in \mathbb{R}^{k} \mid \alpha_{1} \geq \cdots \geq \alpha_{k}\right\} \text { (for decrements). }
$$

Set $\mathcal{A}_{F}$ (resp. $\mathcal{A}_{\bar{F}}$ ) is the largest set of valuations of the $k$ categories for which the ranking of two societies as per Inequality (6) will consider an increment (resp. a decrement) as a normative improvement. The following two propositions establish this formally.

Proposition 2 For any two societies $s$ and $s^{\prime}$, s being obtained from $s^{\prime}$ by an increment as per Definition 1 implies Inequality (6) for all lists of real numbers $\left(\alpha_{1}, \ldots, \alpha_{k}\right) \in$ $\mathcal{A}$, if and only if $\mathcal{A}=\mathcal{A}_{F}$.

Proposition 3 For any two societies $s$ and $s^{\prime}$, s being obtained from $s^{\prime}$ by a decrement as per Definition 2 implies Inequality (6) for all lists of real numbers $\left(\alpha_{1}, \ldots, \alpha_{k}\right) \in$ $\mathcal{A}$, if and only if $\mathcal{A}=\mathcal{A}_{\bar{F}}$.

We now use these propositions to establish the following two theorems, the proof of which makes use of the following technical decomposition result.

Lemma 1 For any society $s$ and any list of valuations $\left(\alpha_{1}, \ldots, \alpha_{k}\right) \in \mathbb{R}^{k}$, we have:

$$
\frac{1}{n} \sum_{h=1}^{k} n_{h}^{s} \alpha_{h}=\alpha_{k}-\sum_{h=1}^{k-1} F(h ; s)\left[\alpha_{h+1}-\alpha_{h}\right],
$$

or equivalently:

$$
\frac{1}{n} \sum_{h=1}^{k} n_{h}^{s} \alpha_{h}=\alpha_{1}+\sum_{h=1}^{k-1} \bar{F}(h ; s)\left[\alpha_{h+1}-\alpha_{h}\right] .
$$


Moreover, for all $t \in\{2, \ldots, k-1\}$, we have:

$$
\frac{1}{n} \sum_{h=1}^{k} n_{h}^{s} \alpha_{h}=\alpha_{t}-\sum_{h=1}^{t-1} F(h ; s)\left[\alpha_{h+1}-\alpha_{h}\right]+\sum_{h=t}^{k-1} \bar{F}(h ; s)\left[\alpha_{h+1}-\alpha_{h}\right] .
$$

The first theorem, which links increment to dominance as per Inequality (6) for all lists of valuations in $\mathcal{A}_{F}$, and to first-order dominance, has been known for quite a long time. (See, for example, Lehmann (1955) or Quirk and Saposnik (1962).) We nonetheless provide a proof of part of it for later use in the proof of the important Theorem 3.

Theorem 1 For any different societies $s$ and $s^{\prime}$, the following three statements are equivalent:

(a) $s$ is obtained from $s^{\prime}$ by means of a finite sequence of increments,

(b) Inequality (6) holds for all $\left(\alpha_{1}, \ldots, \alpha_{k}\right) \in \mathcal{A}_{F}$,

(c) $F(h ; s) \leq F\left(h ; s^{\prime}\right)$ for every category $h \in\{1, \ldots, k-1\}$.

The second theorem is dual to the previous one. It links decrements to both normative dominance for set $\mathcal{A}_{\bar{F}}$ of valuations of the $k$ categories and (anti) first-order dominance. The formal statement of this theorem-whose proof, similar to that of Theorem 1, is left to the reader-is as follows.

Theorem 2 For any different societies $s$ and $s^{\prime}$, the following three statements are equivalent:

(a) $s$ is obtained from $s^{\prime}$ by means of a finite sequence of decrements,

(b) Inequality (6) holds for all $\left(\alpha_{1}, \ldots, \alpha_{k}\right) \in \mathcal{A}_{\bar{F}}$,

(c) $\bar{F}(h ; s) \leq \bar{F}\left(h ; s^{\prime}\right)$ for every category $h \in\{1, \ldots, k-1\}$.

We now turn to Hammond transfers. Paralleling what was established before Propositions 2 and 3, we first seek the conditions on the numerical normative valuations of the categories under which a comparison of two societies based on Inequality (6) would be sensitive to Hammond transfers (as per Definition 3). It turns out that the conditions involve the following subset $\mathcal{H}$ of $\mathbb{R}^{k}$ :

$$
\mathcal{H}=\left\{\left(\alpha_{1}, \ldots, \alpha_{k}\right) \in \mathbb{R}^{k} \mid\left(\alpha_{i}-\alpha_{g}\right) \geq\left(\alpha_{l}-\alpha_{j}\right), \text { for } 1 \leq g<i \leq j<l \leq k\right\}
$$

In words, $\mathcal{H}$ contains all lists of normative categories' valuations that are 'strongly concave' with respect to these categories in the sense that the utility gain from moving from a category to a better one is always larger when moving from categories in the bottom part of the scale than when moving within the upper part of it. The following proposition establishes that set $\mathcal{H}$ of normative categories' valuations is the largest one for which the ranking of two societies based on Inequality (6) would consider favorably the notion of equalization underlying Hammond transfers. 
Proposition 4 For any two societies $s$ and $s^{\prime}$, s being obtained from $s^{\prime}$ by a Hammond transfer as per Definition 3 implies Inequality (6) for all lists of normative valuations $\left(\alpha_{1}, \ldots, \alpha_{k}\right) \in \mathcal{A}$, if and only if $\mathcal{A}=\mathcal{H}$.

The intuition that set $\mathcal{H}$ captures a strong concavity property is, perhaps, better seen through the following proposition, which establishes that $\mathcal{H}$ contains all lists of categories' valuations that are 'single-peaked' in the sense of admitting a largest value before which they are increasing (at a strongly decreasing rate) and after which they are decreasing (at a strongly increasing rate).

Proposition 5 A list of numbers $\left(\alpha_{1}, \ldots, \alpha_{k}\right)$ belongs to $\mathcal{H}$ if and only if there exists a $t \in\{1, \ldots, k\}$ such that $\left(\alpha_{i+1}-\alpha_{i}\right) \geq\left(\alpha_{t}-\alpha_{i+1}\right)$ for all $i \in\{1, \ldots, t-1\}$ (if any) and $\left(\alpha_{i^{\prime}+1}-\alpha_{i^{\prime}}\right) \leq\left(\alpha_{i^{\prime}}-\alpha_{t}\right)$, for all $i^{\prime} \in\{t, \ldots, k-1\}$ (if any).

Two 'peaks' among those identified in Proposition 5 are of particular importance. One is when $t=k$, so that numbers $\left(\alpha_{1}, \ldots, \alpha_{k}\right)$ are increasing (at a strongly decreasing rate) with respect to the categories. In this case, the elements of $\mathcal{H}$ are also in $\mathcal{A}_{F}$. We denote by $\mathcal{A}_{H}=\mathcal{H} \cap \mathcal{A}_{F}$ this set of increasing and strongly concave valuations of the categories. We then have the following immediate (and therefore unproved) corollary of Proposition 5 (applied to $t=k$ ).

Proposition 6 A list of numbers $\left(\alpha_{1}, \ldots, \alpha_{k}\right)$ belongs to $\mathcal{A}_{H}$ if and only if $\left(\alpha_{i+1}-\alpha_{i}\right) \geq$ $\left(\alpha_{k}-\alpha_{i+1}\right)$ holds for all $i \in\{1, \ldots, k-1\}$.

The other extreme of the possible peaks identified in Proposition 5 corresponds to the case where $t=1$, so that the normative valuations $\left(\alpha_{1}, \ldots, \alpha_{k}\right)$ are decreasing (at a strongly increasing rate) with respect to the categories. In this case, the elements of $\mathcal{H}$ are also in $\mathcal{A}_{\bar{F}}$. We denote by $\mathcal{A}_{\bar{H}}=\mathcal{H} \cap \mathcal{A}_{\bar{F}}$ this subset of the set of all strongly concave valuations of the categories that are also decreasing with respect to these categories. We then have the following also immediate (and unproved) corollary of Proposition 5 (applied to $t=1$ ).

Proposition 7 A list of numbers $\left(\alpha_{1}, \ldots, \alpha_{k}\right)$ belongs to $\mathcal{A}_{\bar{H}}$, if and only if it satisfies $\left(\alpha_{i+1}-\alpha_{i}\right) \leq\left(\alpha_{i}-\alpha_{1}\right)$ for all $i \in\{1, \ldots, k-1\}$.

We now establish what we view as the most important result of this paper: $H$ dominance is the implementable test to determine whether one distribution is obtained from another by a finite sequence of either Hammond transfers or increments. The formal statement of this result is as follows.

Theorem 3 For any different societies $s$ and $s^{\prime}$, the following three statements are equivalent:

(a) $s$ is obtained from $s^{\prime}$ by means of a finite sequence of Hammond transfers and/or increments,

(b) Inequality (6) holds for all $\left(\alpha_{1}, \ldots, \alpha_{k}\right) \in \mathcal{A}_{H}$,

(c) $H(h ; s) \leq H\left(h ; s^{\prime}\right)$ for every category $h \in\{1, \ldots, k-1\}$. 
Although a detailed proof of the equivalence of the three statements of Theorem 3 is provided in 'Appendix,' the main arguments are worth presenting here. The fact that Statement (a) implies Statement (b) is an immediate consequence of Propositions 2 and 4. These propositions actually imply that the ranking of two societies based on Inequality (6) is sensitive to Hammond transfers (if the list of valuations $\left(\alpha_{1}, \ldots, \alpha_{k}\right)$ belongs to $\mathcal{H}$ ) and to increments (if $\left(\alpha_{1}, \ldots, \alpha_{k}\right)$ belongs to $\left.\mathcal{A}_{F}\right)$. The proof that Statement (b) implies Statement (c) amounts to verifying that any list of $k$ real numbers $\left(\alpha_{1}^{i}, \ldots, \alpha_{k}^{i}\right)$ defined, for any $i \in\{1, \ldots, k\}$, by:

$$
\begin{aligned}
& \alpha_{h}^{i}=-\left(2^{i-h}\right) \quad \text { for } h=1, \ldots, i \\
& \alpha_{h}^{i}=0 \text { for } h=i+1, \ldots, k
\end{aligned}
$$

belongs to set $\mathcal{A}_{H}$. Indeed, it is apparent from Expression (9) that verifying the inequality:

$$
\sum_{j=1}^{k} n_{j}^{s} \alpha_{j}^{i} \geq \sum_{j=1}^{k} n_{j}^{s^{\prime}} \alpha_{j}^{i}
$$

for any list $\left(\alpha_{1}^{i}, \ldots, \alpha_{k}^{i}\right)$ of real numbers defined as per (23) and (24) for any $i$ is equivalent to the $H$-dominance of $s^{\prime}$ by $s$. Since Inequality (6) holds for all $\left(\alpha_{1}, . ., \alpha_{k}\right)$ in set $\mathcal{A}_{H}$, it must hold in particular for those $\left(\alpha_{1}^{i}, \ldots, \alpha_{k}^{i}\right)$ defined as per (23) and (24) for any $i$. The most difficult proof, that Statement (c) implies Statement (a), is obtained by first noting that if $s$ first-order dominates $s^{\prime}$, then the possibility of going from $s^{\prime}$ to $s$ by a finite sequence of increments is an immediate consequence of Theorem 1. The proof is then constructed under the assumption that $s H$-dominates $s^{\prime}$, but that no first-order dominance exists between the two societies. Hence, there must be categories where the two cumulative distribution functions associated with $s$ and $s^{\prime}$ 'cross.' In that case, we show that a Hammond transfer 'above' the first category for which this crossing occurs can be made in such a way that the new society thereby obtained remains $H$-dominated by $s$. We also show that this Hammond transfer 'brings to naught' at least one of the strict inequalities that distinguish $F(\cdot ; s)$ from $F\left(\cdot ; s^{\prime}\right)$. Hence, if the final distribution $s$ is not reached after this first transfer, then the same procedure can be applied again and again until $s$ is reached. As the number of inequalities that distinguish $F(\cdot, s)$ from $F\left(\cdot, s^{\prime}\right)$ is finite, this proves the implication.

We now state a theorem that is the mirror image of Theorem 3, but with increments replaced by decrements, set $\mathcal{A}_{H}$ by $\mathcal{A}_{\bar{H}}$ and $H$-dominance by $\bar{H}$-dominance. We omit the proof of this theorem whose logic and construction follow those of Theorem $3 .^{4}$

Theorem 4 For any different societies $s$ and $s^{\prime}$, the following three statements are equivalent:

(a) $s$ is obtained from $s^{\prime}$ by means of a finite sequence of Hammond transfers and/or decrements,

(b) Inequality (6) holds for all $\left(\alpha_{1}, \ldots, \alpha_{k}\right) \in \mathcal{A}_{\bar{H}}$,

\footnotetext{
4 The proof is, however, available upon request.
} 
(c) $\bar{H}(h ; s) \leq \bar{H}\left(h ; s^{\prime}\right)$ for every category $h \in\{1, \ldots, k-1\}$.

Theorem 3 (resp. 4) shows that $H$ - (resp. $\bar{H}$-) dominance provides a perfect diagnostic tool to determine the possibility of going from one society to another by a finite sequence of Hammond transfers and/or increments (resp. decrements). But what about the possibility of going from one society to another by Hammond transfers only? It follows clearly from Theorems 3 and 4 that where this possibility exists, the society from which these transfers originate is dominated by the society to which these transfers lead according to both $H$ and $\bar{H}$-dominance. Hence, the possibility of going from one society to another by a finite sequence of Hammond transfers is sufficient for the domination of the former society by the latter as per both $H$ and $\bar{H}$-dominance. The next result establishes the possibility of going from one society to another by a finite sequence of Hammond transfers also necessary for this domination.

Theorem 5 For any different societies $s$ and $s^{\prime}$, the following three statements are equivalent:

(a) $s$ is obtained from $s^{\prime}$ by means of a finite sequence of Hammond transfers,

(b) Inequality (6) holds for all $\left(\alpha_{1}, \ldots, \alpha_{k}\right) \in \mathcal{H}$,

(c) $H(h, s) \leq H\left(h, s^{\prime}\right)$ and $\bar{H}(h, s) \leq \bar{H}\left(h, s^{\prime}\right)$ for every category $h \in\{1, \ldots, k-$ $1\}$.

The proof of this theorem, provided in 'Appendix,' is somewhat different from that of Theorem 3. In effect, it is not constructive. It operates by first establishing an equivalence between Statements (a) and (b) that uses abstract general results on the dual theory for convex cones investigated by Muller and Scarsini (2012) for distributions of continuous variables and developed by Magdalou (2018) for discrete distributions. The second part of the proof establishes a more standard equivalence between Statements (b) and (c). The proof of the latter equivalence makes some use of the following technical result that extends one step further the decomposition (21) of Lemma 1.

Lemma 2 For any society $s$, any list of numbers $\left(\alpha_{1}, \ldots, \alpha_{k}\right) \in \mathbb{R}^{k}$ and any $t \in$ $\{2, \ldots, k-1\}$, we have:

$$
\begin{aligned}
\frac{1}{n} \sum_{h=1}^{k} n_{h}^{s} \alpha_{h}= & \alpha_{t}-\sum_{h=1}^{t-2} H(h ; s)\left[\theta_{h}-\sum_{j=h+1}^{t-1} \theta_{j}\right]-H(t-1 ; s) \theta_{t-1} \\
& +\bar{H}(t ; s) \theta_{t}+\sum_{h=t+1}^{k-1} \bar{H}(h ; s)\left[\theta_{h}-\sum_{j=t}^{h-1} \theta_{j}\right]
\end{aligned}
$$

where $\theta_{h}=\left(\alpha_{h+1}-\alpha_{h}\right)$ for every $h \in\{1, \ldots, k-1\}$.

\section{Sensitivity of the criteria to the grid of categories}

As recognized in classical social choice theory [see, for example, Hammond (1976), Hammond (1976), Deschamps and Gevers (1978), D’Aspremont and Gevers 
(1977) and Sen (1977)], Hammond transfers, when combined with the Pareto principle, are related to the lexicographic extension of the Maximin (or Leximin) criterion for ranking various ordered lists of $n$ numbers. For example, Theorem 4.17 in Blackorby et al. (2005) (ch. 4; p. 123) states that the Leximin criterion is the only monotonically increasing and anonymous ordering of $\mathbb{R}^{n}$ that is strictly sensitive to Hammond transfers. In an analogous vein, Bosmans and Ooghe (2013) and Miyagishima (2010) have shown that the Maximin criterion is the only continuous and Pareto-consistent reflexive and transitive ranking of $\mathbb{R}^{n}$ that is weakly sensitive to Hammond transfers. Since the $H$-dominance criterion coincides, by Theorem 3 , with the possibility of going from the dominated to the dominating society by a finite sequence of Hammond transfers and/or increments - which are nothing more than anonymous Pareto improvementsit is of interest to understand the connection between the $H$-dominance criterion and the Leximin one. We start by defining the latter criterion in the current setting as follows.

Definition 4 Given two societies $s$ and $s^{\prime}$, we say that $s$ dominates $s^{\prime}$ according to the Leximin criterion, which we write $s \succeq_{L} s^{\prime}$, if and only if there exists $i \in\{1, \ldots, k\}$ such that $n_{i}^{s}<n_{i}^{s^{\prime}}$ and $n_{h}^{s}=n_{h}^{s^{\prime}}$ for all integers $h$ such that $1 \leq h<i$ (if any).

It is clear and well known that the Leximin criterion provides a complete and transitive ranking of all societies. By contrast, the $H$-dominance criterion does not provide such a complete ranking of societies as it may leave two societies. Suppose, for example, that there are three categories $(k=3)$ and three agents $(n=3)$ and consider the societies $s$ and $s^{\prime}$ defined by:

$$
n_{1}^{s}=n_{3}^{s}=0, n_{2}^{s}=3
$$

and by:

$$
n_{1}^{s^{\prime}}=1, n_{2}^{s}=0, n_{3}^{s}=2 .
$$

It is clear that $s$ dominates $s^{\prime}$ for the Leximin criterion. However, $s$ and $s^{\prime}$ cannot be compared by the $H$-dominance criterion. The following proposition establishes that the ranking of societies induced by $H$-dominance is a strict subrelation of $\succeq_{L}$.

Proposition 8 Assume that $n>2$. For any two societies $s$ and $s^{\prime}$, if $H(h, s) \leq H\left(h, s^{\prime}\right)$ holds for all categories $h \in\{1, \ldots, k-1\}$, then $s \succeq_{L} s^{\prime}$.

A key difference between our framework and that of classical social choice theory is, of course, the discrete nature of the former. In order to connect the two frameworks, it is useful to examine the sensitivity of the $H$-dominance criterion to the 'level of refinement' of the finite grid over which it is defined. As it turns out, at a suitably high level of grid refinement, the $H$-dominance criterion becomes indistinguishable from the Leximin ordering. There are obviously many ways to refine a given finite grid. In this section, we consider the following notion of $t$-refinement of grid $\mathcal{C}=\{1,2, \ldots, k\}$.

Definition 5 The $t$-refinement of $\operatorname{grid} \mathcal{C}=\{1,2, \ldots, k\}$ for $t \in\{0,1, \ldots\}$, is the set $\mathcal{C}(t)$ defined by $\mathcal{C}(t)=\left\{i / 2^{t}: i=1,2, \ldots,\left(2^{t}\right) k\right\}$. 
Notice that $\mathcal{C}(0)=\mathcal{C}$ so that the initial grid corresponds to 'zero' refinement. The grid obviously becomes finer as $t$ increases, and it is clear that $\mathcal{C}(t) \subset \mathcal{C}(t+1)$ for all $t \in\{0,1, \ldots\}$.

For any society $s$, and any real number $x$ in the interval $(0, k]$, let us denote by $n^{s}(x)$ the (possibly null) number of agents in $s$ who belong to category $x$. As society $s$ is defined on the initial grid $\mathcal{C}$, clearly $n^{s}(x)=0$ for all $x \notin \mathcal{C}$ and $n^{s}(j)=n_{j}^{s}$ for any $j \in \mathcal{C}$. Using these numbers $n^{s}(\cdot)$, and applying the definition of the $H$-curve provided by Eq. (9) to grid $\mathcal{C}(t)$, enables the $t$-refinement of the $H$-curve, denoted for any society $s$ by $H^{t}(\cdot ; s)$, to be defined as follows:

$$
H^{t}(0 ; s)=0
$$

and:

$$
H^{t}\left(\frac{i}{2^{t}} ; s\right)=\frac{1}{n} \sum_{h=1}^{i}\left(2^{i-h}\right) n^{s}\left(\frac{h}{2^{t}}\right), \quad \forall i \in\left\{1,2, \ldots,\left(2^{t}\right) k\right\}
$$

We obviously define the notion of $H$-dominance of a society $s^{\prime}$ by a society $s$ on a $t$ refined grid-referred to as $H^{t}$-dominance — as the fact that a society $s$ has a $H^{t}$-curve nowhere above and somewhere below that of a society $s^{\prime}$. This definition produces a sequence of dominance quasi-orderings indexed by $t$ which, as it turns out, converges to the complete ordering $\succeq_{L}$ when $t$ becomes large.

The first notable effect of such a refinement of the grid is that it reduces the incompleteness of the quasi-ordering of societies induced by the $H$-dominance criterion. Specifically, the following proposition is proved in 'Appendix.'

Proposition 9 For any two societies $s$ and $s^{\prime}$ and any $t \in\{0,1, \ldots\}$, if society $s$ $H^{t}$-dominates society $s^{\prime}$, then society $s H^{t+1}$-dominates society $s^{\prime}$.

Hence, refining the grid increases the discriminating power of the $H^{t}$-dominance criterion. The next theorem establishes that this increase eventually reaches a point where the $H^{t}$-dominance criterion becomes complete and equivalent to the Leximin ordering.

Theorem 6 For any two societies $s$ and $s^{\prime}$ and any initial grid $\mathcal{C}$, the following two statements are equivalent:

(a) There exists an integer $t \in\{0,1, \ldots\}$ such that society $s H^{t}$-dominates society $s^{\prime}$.

(b) $s \succeq_{L} s^{\prime}$.

We conclude this section by pointing out a similar relationship between the $\bar{H}$ dominance criterion and the Lexicographic extension of the Minimax criterion. The Minimax criterion compares alternative lists of $n$ real numbers on the basis of their maximal elements: The lower the maximal element, the better the list. The lexicographic extension of the Minimax criterion-anti-Leximax for short-extends the principle to the second maximal element, and to the third and so on when the maximal, the second maximal and so on of two lists are identical. While the Leximin and 
the Maximin criteria can be seen as ethically favoring the 'worst off,' the Minimax or the anti-Leximax criteria disfavor the 'best off.'

The fact that $\bar{H}$-dominance converges to the anti-Leximax criterion and $H$ dominance converges to the Leximin one when the grid becomes sufficiently fine has obvious, but important, implication for the criterion defined in the preceding subsection as the intersection of the $H$ - and the $\bar{H}$-dominance criteria. This intersection of $H$ - and $\bar{H}$-dominance must converge to the intersection of the Leximin and the anti-Leximax criteria when the grid becomes sufficiently fine. This result is of course in line with Gravel et al. (2019) who shows that the intersection of the Leximin and the anti-Leximax criteria is the smallest transitive relation that is strictly sensitive to Hammond transfers.

\section{Empirical illustration}

We now provide a brief illustration of our criteria for comparing distributions of individuals' self-reported levels of life satisfaction — or happiness — in a selected number of countries in 2014. These distributions of self-reported life satisfactions feed the yearly UN World Happiness Reports. (See https://worldhappiness.report.) The reported life satisfaction variable results from the World Gallup Poll who asks to a sample of respondents 'to think of a ladder, with the best possible life for them being a 10, and the worst possible life being a 0.' A ladder like this is sometimes called a Cantril ladder. The samples surveyed by the Gallup polls are representative, but somewhat small (between 1000 and 1500 individuals per country). The data used herein are taken from 2014 and have been obtained from the Pew Research Center (https://www. pewresearch.org). We feel that reported happiness, even when subjectively located on an 'equally spaced' Cantril ladder, conveys at best an ordinal information on the underlying psychological trait that it measures, whatever this is. Like many readers of the happiness measurement literature, we may actually be doubtful that the satisfaction reported by an individual who locates herself (himself) on a Cantril is comparable across individuals. But clearly, the Cantril ladder is. It is therefore of some interest to look at the distributions of those individuals' localizations and to compare them across countries.

The distributions of self-reported life satisfaction (ranging from 0 to 10) in the compared countries (we took the 29 most populated countries for which the data were available in 2014) decreasingly ordered by the Leximin criterion are provided in Table 1. Observe that the Leximin ranking of countries is quite different from that based on the average value of the happiness score often discussed and commented in the literature. It also differs from the ranking based on the median self-reported category that is sometimes considered to be a natural measure of the 'central tendency' of the distribution of an ordinal variable. [See, for example, Allison and Foster (2004).] To some extent, the difference between the Leximin ranking of countries and those based on the median or the average results from the 'dictatorship of the worst-off' effect. Indeed, in many developing countries (and notably in Indonesia, Bangladesh, China, India and Nigeria), there is nobody who declares a zero level of life satisfaction. These 


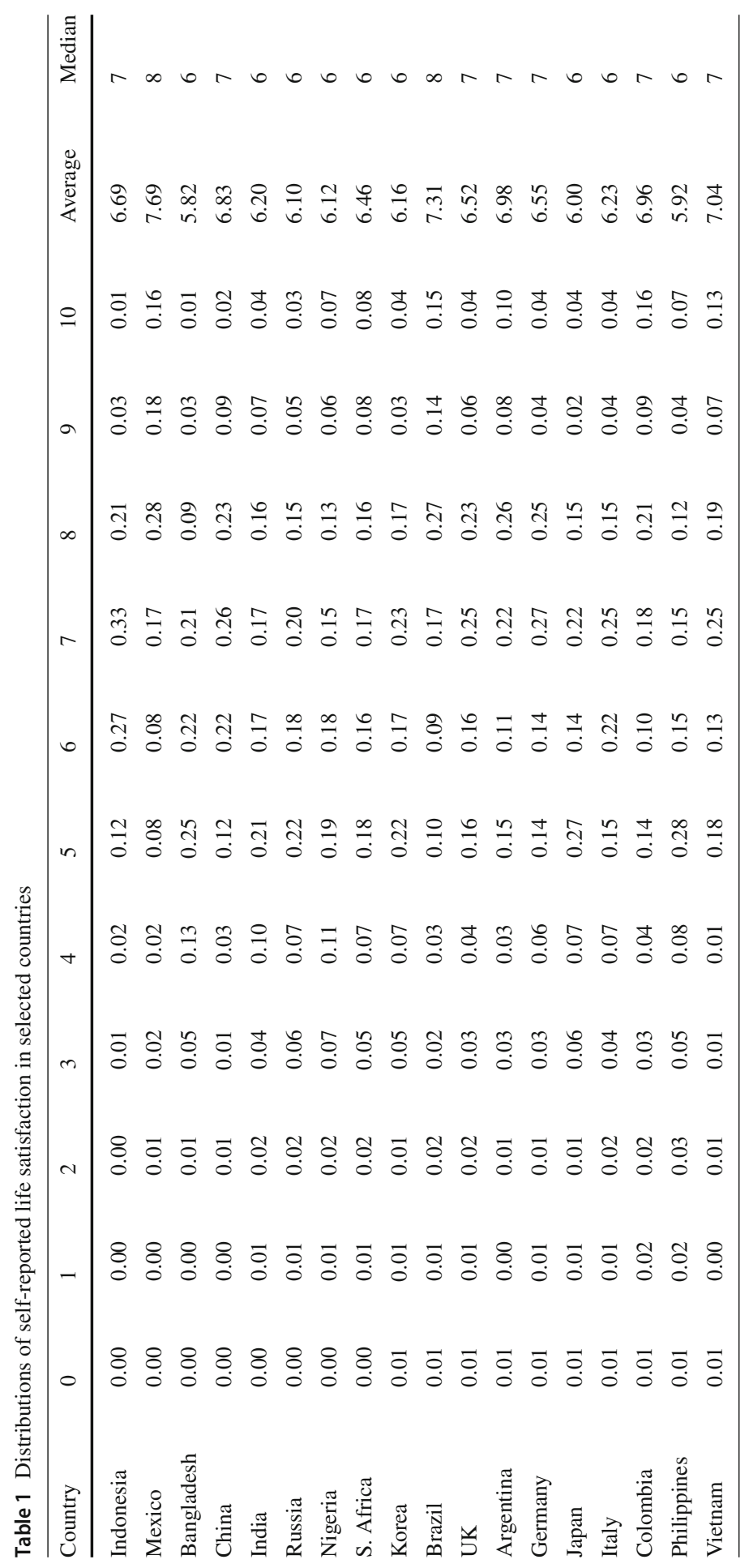




\begin{tabular}{|c|c|}
\hline 8 & 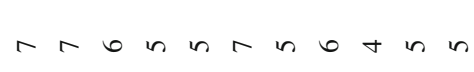 \\
\hline & त̂ \\
\hline & 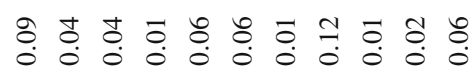 \\
\hline & 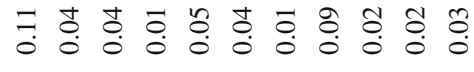 \\
\hline & 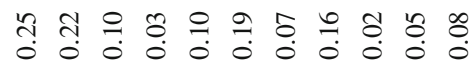 \\
\hline & तิ तี \\
\hline & 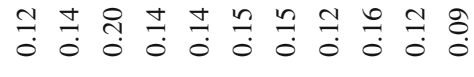 \\
\hline & 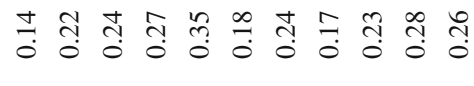 \\
\hline & 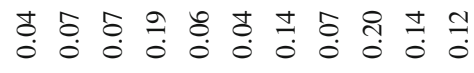 \\
\hline & 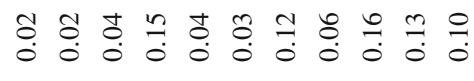 \\
\hline & 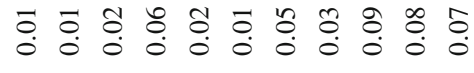 \\
\hline & 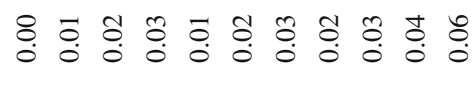 \\
\hline & 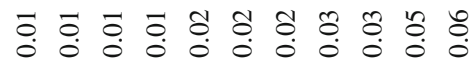 \\
\hline & 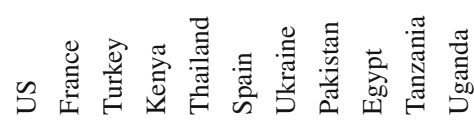 \\
\hline
\end{tabular}


countries are therefore ranked favorably by the Leximin criterion. The somewhat weak correlation between the ranking of countries based on pecuniary indicators (such as per capita GDP) and that based on reported life satisfaction is also worth noticing.

We now provide the rankings of distributions of these self-reported life satisfactions by means of Hasse diagrams based on the three criteria discussed herein. On every such a diagram, in which countries are again vertically ordered as per the Leximin criterion, dominance between two countries is indicated by a non-increasing sequence of lines and arcs connecting the dominating to the dominated country.

The (somewhat indecisive) Fig. 1 depicts the Hasse diagram of the first-order dominance ranking of the distributions of self-reported life satisfaction assessment. First-order dominance happens indeed to be mildly discriminant in the present case since it enables the conclusive ranking of only 148 pairs of countries out of the 406 possible such pairs, a rate of comparability of about $36 \%$. The ranking shows the strong position of Mexico (who dominates all countries but Indonesia, China and Vietnam) and the weak one of Egypt (dominated by all countries but Tanzania, Uganda, Kenya and Ukraine). Countries like Bangladesh and Indonesia, on the other hand, happen to be hardly comparable with others. Indonesia can only be connected with two countries that it dominates: Bangladesh and Egypt. Bangladesh, on the other hand, is dominated by both Indonesia and Mexico and dominates Egypt. It is not comparable with any other country.

Adding equality considerations in the form of Hammond transfers to the increments that underlie first-order dominance substantially increases substantially the fraction of conclusive rankings, as shown in Fig. 2. In effect, $H$-dominance enables the conclusive ranking of 329 pairs of countries (a bit more than $81 \%$ of all the possible pairs). Bangladesh and Indonesia-who could not be compared to most countries on the basis of first-order dominance-see their position greatly enhanced as a result of switching to $H$-dominance. Indonesia $H$-dominates indeed all countries but Mexico. As for Bangladesh, while it obviously remains dominated by Mexico and Indonesia, it dominates all other countries but Vietnam and the USA. It is noteworthy that these two countries are those who are the least comparable to others in terms of $H$-dominance. While their average (and median) level of reported life satisfaction is somewhat high (above 7), it happens to be somewhat unequally distributed.

Are there countries where reported life satisfaction is more equally distributed than in others in the sense of Hammond transfers only? It turns out that there are! Figure 3 shows the Hasse diagram connecting the countries that can be compared with at least one other by the intersection of $H$ and $\bar{H}$-dominance. Observe that only one country, Mexico, is left out of this diagram. This is not surprising since this country dominates almost all others by first-order dominance only. It can therefore not be compared by the intersection of $H$ and $\bar{H}$-dominance. Not surprisingly, Indonesia and Bangladesh appear to be top performers in terms of happiness equalization. Pakistan, on the other hand, appears to be a country where happiness is unequally distributed as is, to a lesser extent, Spain. 


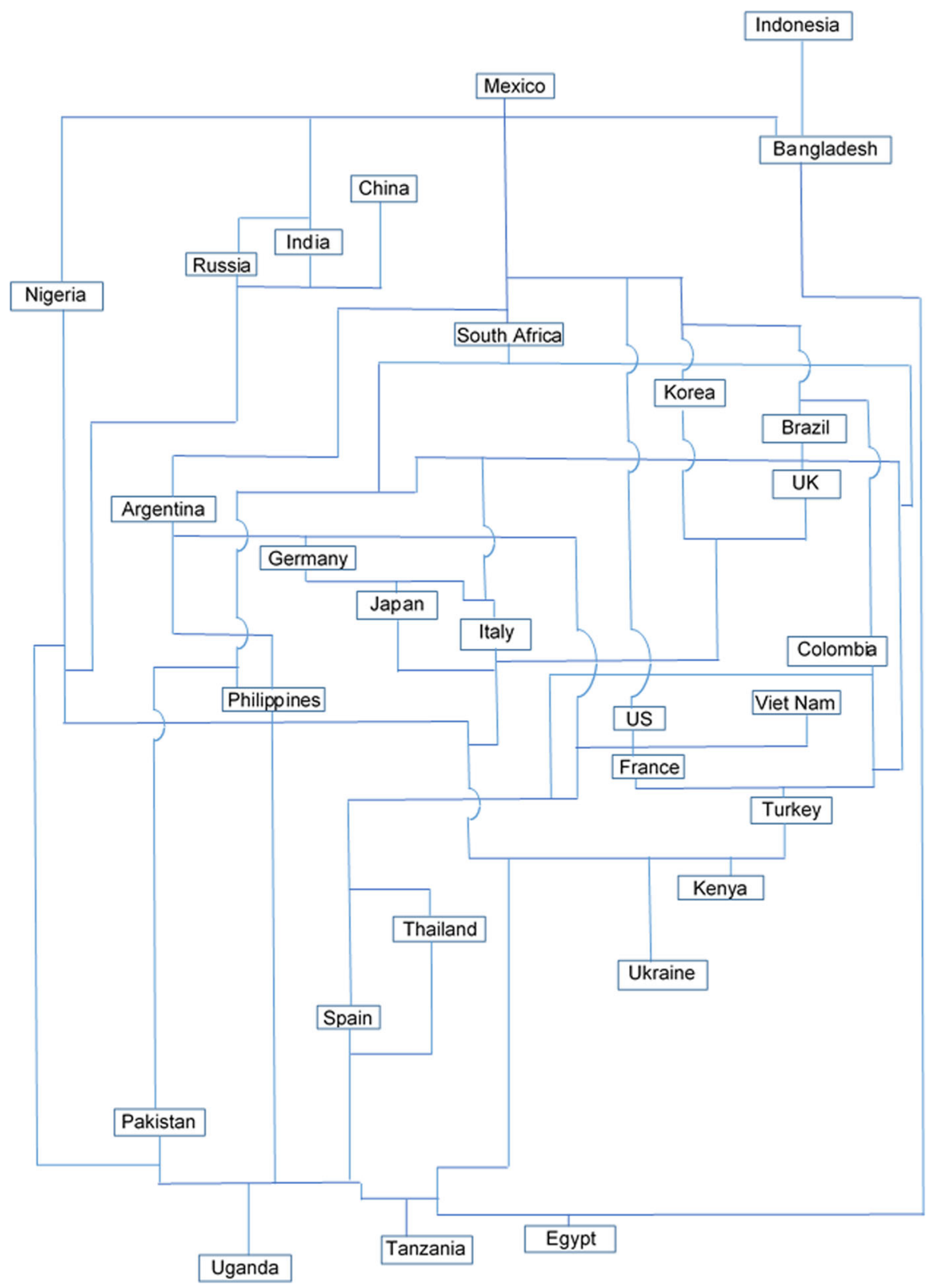

Fig. 1 First-order dominance chart 


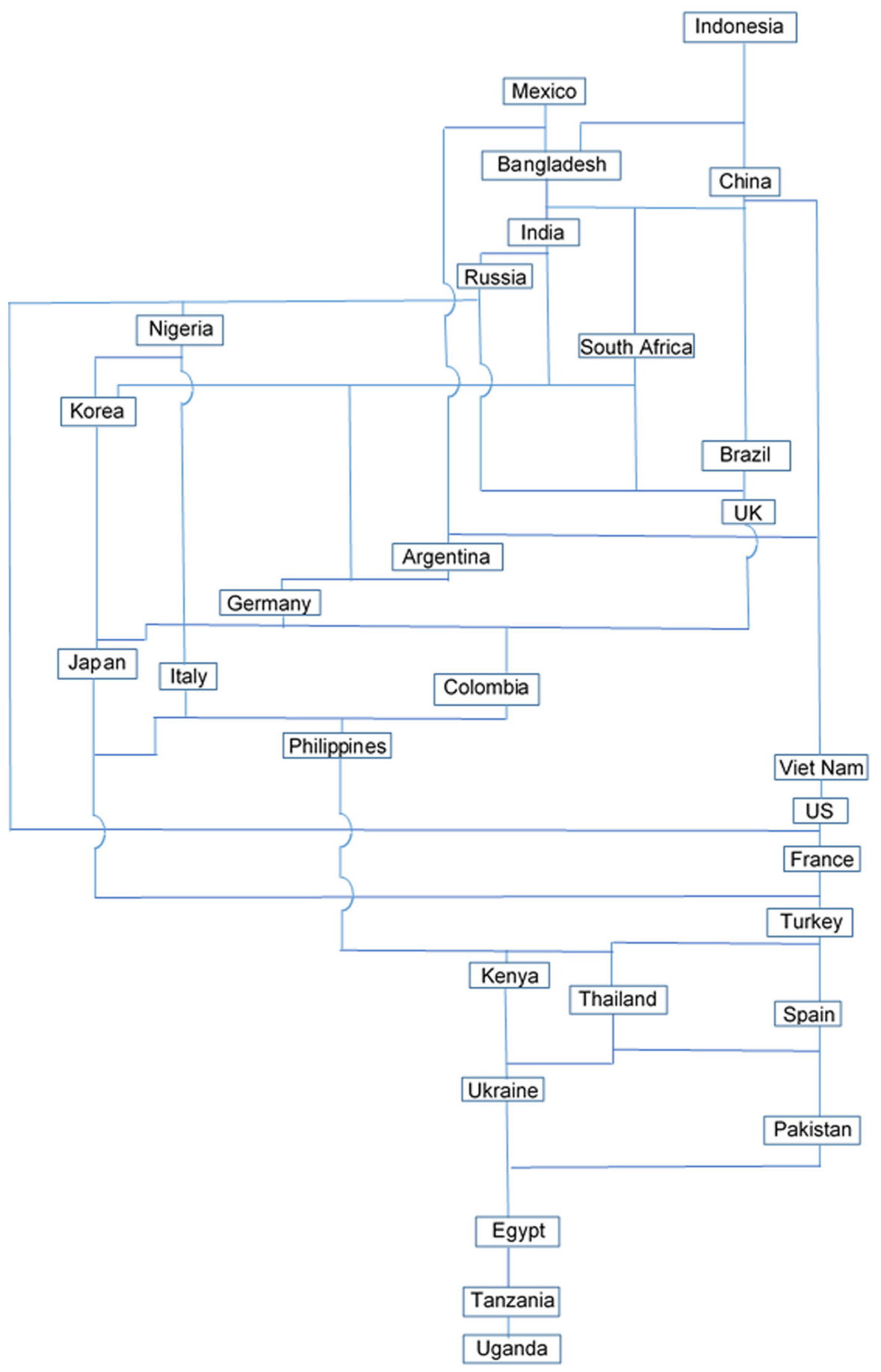

Fig. $2 H$-dominance chart 


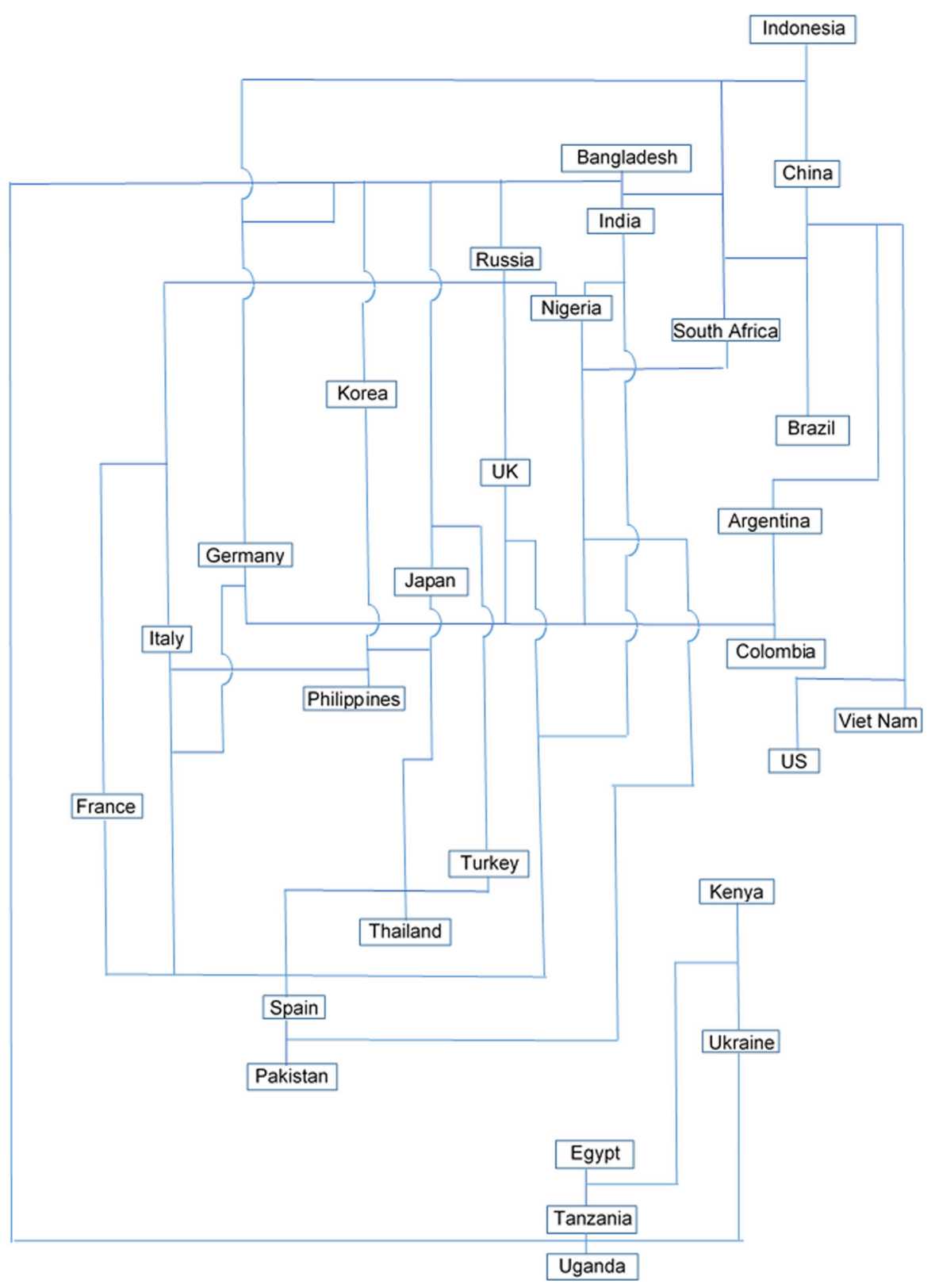

Fig. 3 Dominance ranking based on happiness-equality only 


\section{Conclusion}

The paper has laid the groundwork for comparing distributions of an ordinal variable that takes finitely many values. The crux of our analysis is an easy-to-use criterion, called $\mathrm{H}$-dominance. This criterion can be viewed as the analogue, for comparing distributions of an ordinally measurable attribute, of the generalized Lorenz curve used for comparing distributions of a cardinally measurable one. It is well known [see, for example, Shorrocks (1983)] that one distribution of a cardinally measurable attribute dominates another for the generalized Lorenz criterion if and only if it is possible to go from the dominated distribution to the dominating one by a finite sequence of increments in the attribute and/or Pigou-Dalton transfers. The main result of this paper-Theorem 3-establishes an analogous result for the $H$-dominance criterion. We show that the latter criterion ranks two distributions of an attribute in the same way as would going from the dominated to the dominating distribution by a finite sequence of increments and/or Hammond transfers. We also identified a dual $\bar{H}$ dominance criterion that ranks two distributions in the same way as would going from the dominated to the dominating distribution by a finite sequence of decrements and/or Hammond transfers of the attribute. We also show that the intersection of the $H$-and the $\bar{H}$-dominance criteria coincides with the possibility of going from the dominated to the dominating distribution by a finite sequence of Hammond transfers only.

As illustrated with the distributions of self-declared indices of life assessment, the $H$-dominance criterion, and the Hammond principle of transfers that justifies it along with increments, appears to be a useful tool for comparing distributions of a variable that cannot be meaningfully transferred à la Pigou-Dalton. Not only is the $H$-dominance criterion justified by clear and meaningful elementary transformations, it also has the advantage of being applicable to a much wider class of situations than the widely discussed criterion proposed by Allison and Foster (2004). The latter is limited to distributions that have the same median and is not associated with clear and meaningful elementary transformations. As shown with the empirical illustration, the criteria characterized in this paper are quite capable of comparing, even from the pure viewpoint of (Hammond-based) equality considerations, distributions with different medians.

We also emphasize that while we sought the criteria and transfers principles discussed here mainly so as to apply them to distributions of an ordinally measurable attribute, they can also be applied to a cardinally measurable attribute if the strong egalitarian flavor of Hammond transfers is deemed appropriate for that purpose.

Among the many possible extensions of the approach developed in this paper, two strike us as particularly important. First, since the $H$-criterion is incomplete in the discrete setting, it would be interesting to obtain simple inequality indices that are compatible with Hammond transfers and, therefore, with the intersection of $H$-and $\bar{H}$-dominance. We believe that obtaining an axiomatic characterization of a family of such indices would not be too difficult. A good starting point would be to consider indices that can be written as per Expression (6) for some suitable choice of lists $\left(\alpha_{1}, \ldots, \alpha_{k}\right)$ of real numbers. A second extension, obviously more difficult, would be to consider multidimensional attributes. 


\section{A Appendix: Proofs}

\section{A.1 Proposition 1}

Let $s$ and $s^{\prime}$ be two societies such that $F(i ; s) \leq F\left(i ; s^{\prime}\right)$ holds for all $i \in\{1, \ldots, k-1\}$. It follows that $F(1 ; s) \leq F\left(1 ; s^{\prime}\right)$ and:

$$
\sum_{h=1}^{i-1}\left(2^{i-h-1}\right) F(h ; s)+F(i ; s) \leq \sum_{h=1}^{i-1}\left(2^{i-h-1}\right) F\left(h ; s^{\prime}\right)+F\left(i ; s^{\prime}\right), \forall i \in\{2, \ldots, k\}
$$

so that, thanks to Expressions (10) and (11), $H(i ; s) \leq H\left(i ; s^{\prime}\right)$ holds for all $i \in$ $\{1, \ldots, k-1\}$. To establish the $\bar{H}$-dominance of $s$ by $s^{\prime}$, it suffices to notice that the requirement $F(i ; s) \leq F\left(i ; s^{\prime}\right)$ for all $i \in\{1, \ldots, k-1\}$ can alternatively be written as $\bar{F}(i ; s) \geq \bar{F}\left(i, s^{\prime}\right)$ for all $i \in\{1, \ldots, k-1\}$. That implies $\bar{F}(k-1 ; s) \geq \bar{F}\left(k-1 ; s^{\prime}\right)$ and:

$$
\begin{aligned}
& \sum_{h=i+1}^{k-1}\left(2^{h-i-1}\right) \bar{F}(h ; s)+\bar{F}(i ; s) \\
& \geq \sum_{h=i+1}^{k-1}\left(2^{h-i-1}\right) \bar{F}\left(h ; s^{\prime}\right)+\bar{F}\left(i ; s^{\prime}\right), \quad \forall i \in\{1, \ldots, k-2\}
\end{aligned}
$$

so that, thanks to Expressions (15) and (16), $\bar{H}\left(i ; s^{\prime}\right) \leq H(i ; s)$ for all $i \in\{1, \ldots, k-$ $1\}$.

\section{A.2 Propositions 2 and 3}

For Proposition 2, let $s$ be a society obtained from $s^{\prime}$ by an increment. By Definition 1, there exists some $j \in\{1, \ldots, k-1\}$ such that we have $n_{j}^{s}=n_{j}^{s^{\prime}}-1$ and $n_{j+1}^{s}=$ $n_{j+1}^{s^{\prime}}+1$ and that, for all $h \in\{1, \ldots, k\}$ such that $h \neq j, j+1$, we have $n_{h}^{s}=n_{h}^{s^{\prime}}$. It follows that:

$$
\sum_{h=1}^{k} n_{h}^{s} \alpha_{h}=\sum_{h=1}^{k} n_{h}^{s^{\prime}} \alpha_{h}+\left(\alpha_{j+1}-\alpha_{j}\right)
$$

Thus, inequality $\sum_{h=1}^{k} n_{h}^{s} \alpha_{h} \geq \sum_{h=1}^{k} n_{h}^{s^{\prime}} \alpha_{h}$ holds if and only if $\left(\alpha_{j+1}-\alpha_{j}\right) \geq 0$. As this inequality must hold for any $j \in\{1, \ldots, k-1\}$, this completes the proof of Proposition 2. The argument for Proposition 3 is similar (with Definition 1 replaced by Definition 2).

\section{A.3 Lemma 1}

Observe first that: 


$$
\sum_{h=1}^{k} n_{h}^{s} \alpha_{h}=\left\{\begin{array}{c}
n_{1}^{s} \alpha_{1} \\
+n_{2}^{s} \alpha_{2} \\
+\cdots \\
+n_{k}^{s} \alpha_{k}
\end{array}\right.
$$

or equivalently:

$$
\sum_{h=1}^{k} n_{h}^{s} \alpha_{h}=\left\{\begin{array}{l}
\quad n_{1}^{s} \alpha_{1} \\
+n_{2}^{s} \alpha_{1}+n_{2}^{s}\left[\alpha_{2}-\alpha_{1}\right] \\
+n_{3}^{s} \alpha_{1}+n_{3}^{s}\left[\alpha_{2}-\alpha_{1}\right]+n_{3}^{s}\left[\alpha_{3}-\alpha_{2}\right] \\
+\cdots \\
+n_{k}^{s} \alpha_{1}+n_{k}^{s}\left[\alpha_{2}-\alpha_{1}\right]+n_{k}^{s}\left[\alpha_{3}-\alpha_{2}\right]+\cdots n_{k}^{s}\left[\alpha_{k}-\alpha_{k-1}\right]
\end{array}\right.
$$

hence:

$$
\sum_{h=1}^{k} n_{h}^{s} \alpha_{h}=\left\{\begin{array}{l} 
\\
n \alpha_{1} \\
+\left(n-n_{1}^{s}\right)\left[\alpha_{2}-\alpha_{1}\right] \\
+\left[n-\left(n_{1}^{s}+n_{2}^{s}\right)\right]\left[\alpha_{3}-\alpha_{2}\right] \\
+\cdots \\
+\left[n-\sum_{h=1}^{k-1} n_{h}^{s}\right]\left[\alpha_{k}-\alpha_{k-1}\right]
\end{array}\right.
$$

from which one obtains:

$$
\begin{aligned}
\frac{1}{n} \sum_{h=1}^{k} n_{h}^{s} \alpha_{h} & =\left[\alpha_{1}+\left(\alpha_{k}-\alpha_{1}\right)\right]-\sum_{h=1}^{k-1} F(h ; s)\left[\alpha_{h+1}-\alpha_{h}\right] \\
& =\alpha_{k}-\sum_{h=1}^{k-1} F(h ; s)\left[\alpha_{h+1}-\alpha_{h}\right],
\end{aligned}
$$

as required by Eq. (19). Now, by reconsidering Eq. (29) and recalling that $\bar{F}(i ; s)=$ $1-F(i ; s)=\left(n-\sum_{h=1}^{i} n_{h}^{s}\right) / n$ for every $i \in \mathcal{C}$, one immediately obtains Eq. (20). We must now establish Eq. (21). For this sake, one can notice that, for any $t \in$ $\{2, \ldots, k-1\}$, one has:

$$
\sum_{h=1}^{k} n_{h}^{s} \alpha_{h}=\sum_{h=1}^{t} n_{h}^{s} \alpha_{h}+\sum_{h=t+1}^{k} n_{h}^{s} \alpha_{h} .
$$

If one successively decomposes the two terms on the right hand of (32), one obtains for the first one:

$$
\sum_{h=1}^{t} n_{h}^{s} \alpha_{h}=\left\{\begin{array}{l}
\quad n_{1}^{s} \alpha_{1} \\
+n_{2}^{s} \alpha_{1}+n_{2}^{s}\left[\alpha_{2}-\alpha_{1}\right] \\
+n_{3}^{s} \alpha_{1}+n_{3}^{s}\left[\alpha_{2}-\alpha_{1}\right]+n_{3}^{s}\left[\alpha_{3}-\alpha_{2}\right] \\
+\cdots \\
+n_{t}^{s} \alpha_{1}+n_{t}^{s}\left[\alpha_{2}-\alpha_{1}\right]+n_{i}^{s}\left[\alpha_{3}-\alpha_{2}\right]+\cdots n_{t}^{s}\left[\alpha_{t}-\alpha_{t-1}\right]
\end{array}\right.
$$


One has therefore:

$$
\sum_{h=1}^{t} n_{h}^{s} \alpha_{h}=\left\{\begin{aligned}
& \left(\sum_{h=1}^{t} n_{h}^{s}\right) \alpha_{1} \\
+ & {\left[\sum_{h=1}^{t} n_{h}^{s}-n_{1}^{s}\right]\left[\alpha_{2}-\alpha_{1}\right] } \\
+ & {\left[\sum_{h=1}^{t} n_{h}^{s}-\left(n_{1}^{s}+n_{2}^{s}\right)\right]\left[\alpha_{3}-\alpha_{2}\right] } \\
+ & \cdots \\
+ & {\left[\sum_{h=1}^{t} n_{h}^{s}-\sum_{h=1}^{t-1} n_{h}^{s}\right]\left[\alpha_{t}-\alpha_{t-1}\right] }
\end{aligned}\right.
$$

or equivalently:

$$
\frac{1}{n} \sum_{h=1}^{t} n_{h}^{s} \alpha_{h}=\left(\frac{1}{n} \sum_{h=1}^{t} n_{h}^{s}\right) \alpha_{t}-\sum_{h=1}^{t-1} F(h ; s)\left[\alpha_{h+1}-\alpha_{h}\right] .
$$

For the second term of (32), the successive decomposition yields:

$$
\sum_{h=t+1}^{k} n_{h}^{s} \alpha_{h}=\left\{\begin{array}{l}
\quad n_{t+1}^{s} \alpha_{t+1} \\
+n_{t+2}^{s} \alpha_{t+1}+n_{t+2}^{s}\left[\alpha_{t+2}-\alpha_{t+1}\right] \\
+n_{t+3}^{s} \alpha_{t+1}+n_{t+3}^{s}\left[\alpha_{t+2}-\alpha_{t+1}\right]+n_{t+3}^{s}\left[\alpha_{t+3}-\alpha_{t+2}\right] \\
+\cdots \\
+n_{k}^{s} \alpha_{t+1}+n_{k}^{s}\left[\alpha_{t+2}-\alpha_{t+1}\right]+n_{k}^{s}\left[\alpha_{t+3}-\alpha_{t+2}\right]+\cdots n_{k}^{s}\left[\alpha_{k}-\alpha_{k-1}\right],
\end{array}\right.
$$

This can be written as:

$$
\sum_{h=t+1}^{k} n_{h}^{s} \alpha_{h}=\left\{\begin{aligned}
& \left(\sum_{h=t+1}^{k} n_{h}^{s}\right) \alpha_{t+1} \\
+ & \left(\sum_{h=t+2}^{k} n_{h}^{s}\right)\left[\alpha_{t+2}-\alpha_{t+1}\right] \\
+ & \left(\sum_{h=t+3}^{k} n_{h}^{s}\right)\left[\alpha_{t+3}-\alpha_{t+2}\right] \\
+ & \cdots \\
+ & n_{k}^{s}\left[\alpha_{k}-\alpha_{k-1}\right]
\end{aligned}\right.
$$

or equivalently:

$$
\frac{1}{n} \sum_{h=t+1}^{k} n_{h}^{s} \alpha_{h}=\left(\frac{1}{n} \sum_{h=t+1}^{k} n_{h}^{s}\right) \alpha_{t+1}+\sum_{h=t+1}^{k-1} \bar{F}(h ; s)\left[\alpha_{h+1}-\alpha_{h}\right] .
$$

By summing Eqs. (33) and (34), one concludes that:

$$
\begin{aligned}
\frac{1}{n} \sum_{h=1}^{k} n_{h}^{s} \alpha_{h}= & \left(\frac{1}{n} \sum_{h=1}^{t} n_{h}^{s}\right) \alpha_{t}+\left(\frac{1}{n} \sum_{h=t+1}^{k} n_{h}^{s}\right) \alpha_{t+1} \\
& -\sum_{h=1}^{t-1} F(h ; s)\left[\alpha_{h+1}-\alpha_{h}\right]+\sum_{h=t+1}^{k-1} \bar{F}(h ; s)\left[\alpha_{h+1}-\alpha_{h}\right] .
\end{aligned}
$$


This equality can be further simplified, by observing that:

$$
\begin{aligned}
\left(\frac{1}{n} \sum_{h=1}^{t} n_{h}^{s}\right) \alpha_{t}+\left(\frac{1}{n} \sum_{h=t+1}^{k} n_{h}^{s}\right) \alpha_{t+1} & =\frac{1}{n}\left(n-\sum_{h=t+1}^{k} n_{h}^{s}\right) \alpha_{t}+\left(\frac{1}{n} \sum_{h=t+1}^{k} n_{h}^{s}\right) \alpha_{t+1} \\
& =\alpha_{t}+\bar{F}(t ; s)\left[\alpha_{t+1}-\alpha_{t}\right] .
\end{aligned}
$$

Equation (21) is then obtained from the reintroduction of (36) into (35).

\section{A.4 Theorem 1}

The equivalence between Statements (a) and (c) of this theorem is well known in the literature. We therefore only prove the equivalence between Statements (b) and (c). Using Eq. (19) of Lemma 1, one has:

$$
\frac{1}{n}\left[\sum_{h=1}^{k} n_{h}^{s} \alpha_{h}-\sum_{h=1}^{k} n_{h}^{s^{\prime}} \alpha_{h}\right]=\sum_{h=1}^{k-1}\left[F\left(h ; s^{\prime}\right)-F(h ; s)\right]\left[\alpha_{h+1}-\alpha_{h}\right] .
$$

Hence, if $F\left(h ; s^{\prime}\right)-F(h ; s) \geq 0$ for every $h \in \mathcal{C}$ and $\left(\alpha_{1}, \ldots, \alpha_{k}\right) \in \mathcal{A}_{F}$, then $\sum_{h=1}^{k} n_{h}^{s} \alpha_{h} \geq \sum_{h=1}^{k} n_{h}^{s^{\prime}} \alpha_{h}$. To establish the converse implication, define, for every $i \in\{1, \ldots, k-1\}$, the list of $k$ numbers $\alpha^{i}=\left(\alpha_{1}^{i}, \ldots, \alpha_{k}^{i}\right)$ to be such that $\alpha_{h}^{i}=0$ for $h=1, \ldots, i$ and $\alpha_{h}^{i}=1$ for $h \in\{i+1, \ldots, k\}$. We note that $\alpha^{i} \in \mathcal{A}_{F}$ for any $i \in\{1, \ldots, k-1\}$. If Inequality (6) holds for all lists of numbers $\left(\alpha_{1}, \ldots, \alpha_{k}\right) \in$ $\mathcal{A}_{F}$, then one must have $\sum_{h=1}^{k} n_{h}^{s} \alpha_{h}^{i} \geq \sum_{h=1}^{k} n_{h}^{s^{\prime}} \alpha_{h}^{i}$ for any $i \in\{1, \ldots, k-1\}$ or, equivalently:

$$
\left(n-\sum_{h=1}^{i} n_{h}^{s}\right)=\sum_{h=i+1}^{k} n_{h}^{s} \geq \sum_{h=i+1}^{k} n_{h}^{s^{\prime}}=\left(n-\sum_{h=1}^{i} n_{h}^{s^{\prime}}\right),
$$

which, in turn, is equivalent to $\sum_{h=1}^{i} n_{h}^{s} \leq \sum_{h=1}^{i} n_{h}^{s^{\prime}}$, as required.

\section{A.5 Proposition 4}

Suppose that society $s$ has been obtained from society $s^{\prime}$ by means of a Hammond transfer as per Definition 3. This means that there are categories $1 \leq g<i \leq j<$ $l \leq k$ for which one has:

$$
\sum_{h=1}^{k} n_{h}^{s} \alpha_{h}=\sum_{h=1}^{k} \alpha_{h} n_{h}^{s^{\prime}}-\alpha_{g}+\alpha_{i}+\alpha_{j}-\alpha_{l}
$$

Hence, if Inequality (6) holds for $s$ and $s^{\prime}$, one have $\sum_{h=1}^{k} n_{h}^{s} \alpha_{h}-\sum_{h=1}^{k} n_{h}^{s^{\prime}} \alpha_{h}=$ $\left(\alpha_{i}-\alpha_{g}\right)-\left(\alpha_{l}-\alpha_{j}\right) \geq 0$ for all categories $1 \leq g<i \leq j<l \leq k$, which is precisely the definition of the set $\mathcal{H}$. 


\section{A.6 Proposition 5}

Assume that the list of numbers $\left(\alpha_{1}, \ldots, \alpha_{k}\right)$ belongs to $\mathcal{H}$ and, therefore, satisfies $\alpha_{i}-\alpha_{g} \geq \alpha_{l}-\alpha_{j}$ for all $1 \leq g<i \leq j<l \leq k$. This implies in particular that $\alpha_{i+1}-$ $\alpha_{i} \geq \alpha_{k}-\alpha_{i+1}$ for any $i \in\{1,2, \ldots, k-2\}$. Let $t=\min \left\{i=1, \ldots, k \mid \alpha_{i+1}-\alpha_{i} \leq 0\right\}$ (using the convention that $\alpha_{k+1}=\alpha_{k}$ ). Such a $t$ clearly exists under this convention, because $k \in\left\{i=1, \ldots, k \mid \alpha_{i+1}-\alpha_{i} \leq 0\right\}$. If $t=k$, then the fact that $\alpha_{i+1}-\alpha_{i} \geq$ $\alpha_{k}-\alpha_{i+1}$ holds for any $i \in\{1,2, \ldots, k-2\}$ implies that $\alpha_{i+1}-\alpha_{i} \geq \alpha_{t}-\alpha_{i+1}$ for all $i \in\{1,2, \ldots, t-1\}$ and (trivially) that $\alpha_{i^{\prime}+1}-\alpha_{i^{\prime}} \leq \alpha_{i^{\prime}}-\alpha_{t}$ holds for all $i^{\prime} \in\{t, \ldots, k-1\}=\varnothing$. Notice that if $t=k$, then one has $\alpha_{i+1}-\alpha_{i} \geq \alpha_{k}-\alpha_{i+1}>0$ for any $i \in\{1,2, \ldots, k-2\}$. (The alphas are increasing with respect to the categories.) If $t=1$, then the set $\{i=1,2, \ldots, t-1\}$ is empty so that one must simply verify that $\alpha_{i^{\prime}+1}-\alpha_{i^{\prime}} \leq \alpha_{i^{\prime}}-\alpha_{1}$, for $i^{\prime} \in\{1, \ldots, k-1\}$. But this results immediately from the definition of $t$ (if $i^{\prime}=1$ ) or from applying the requirement that $\alpha_{i}-\alpha_{g} \geq \alpha_{l}-\alpha_{j}$ for all $1 \leq g<i \leq j<l \leq k$ to the particular case where $g=1, i=j=i^{\prime}$ $>1$ and $l=i^{\prime}+1$ (otherwise). Notice that if $t=1$, then one has by definition that $0 \geq \alpha_{2}-\alpha_{1} \geq \alpha_{j}-\alpha_{j-1}$ for every $j \in\{3, \ldots, k\}$ so that the alphas are decreasing with the categories. Assume now that $t \in\{2, \ldots, k-1\}$. We must check first that $\alpha_{i+1}-\alpha_{i} \geq \alpha_{t}-\alpha_{i+1}$ for all $i=1,2, \ldots, t-1$. The case where $i=t-1$ is proved by observing that, by definition of $t$, one has $\alpha_{t}-\alpha_{t-1}>0=\alpha_{t}-\alpha_{t}$. The case where $i<t-1$ (if any) is proved by applying the statement $\alpha_{i}-\alpha_{g} \geq \alpha_{l}-\alpha_{j}$ for all $1 \leq g<i \leq j<l \leq k$ to the particular case where $g=i \in\{1, \ldots, t-2\}$ $i=j=i+1$ and $l=t$. To check that the inequality $\alpha_{i^{\prime}+1}-\alpha_{i^{\prime}} \leq \alpha_{i^{\prime}}-\alpha_{t}$ holds for all $i^{\prime} \in\left\{t^{\prime}, \ldots, k-1\right\}$, simply observe that, for $i^{\prime}=t$, the inequality is obtained from the very definition of $t$ and, for $i^{\prime}>t$, it results from applying the fact that $\alpha_{i}-\alpha_{g} \geq \alpha_{l}-\alpha_{j}$ for all $1 \leq g<i \leq j<l \leq k$ to the particular case where $g=t$, $i=j=i^{\prime}$ and $l=i^{\prime}+1$.

Conversely, consider any list $\left(\alpha_{1}, \ldots, \alpha_{k}\right)$ for which there exists a $t \in\{1, \ldots, k\}$ such that:

$$
\alpha_{i+1}-\alpha_{i} \geq \alpha_{t}-\alpha_{i+1}
$$

holds for all $i \in\{1,2, \ldots, t-1\}$ (if any) and:

$$
\alpha_{i^{\prime}+1}-\alpha_{i^{\prime}} \leq \alpha_{i^{\prime}}-\alpha_{t}
$$

holds for all $i^{\prime} \in\{t, \ldots, k-1\}$ (if any). Notice that applying Inequality (40) to $i=t-1$ implies that $\alpha_{t}-\alpha_{t-1} \geq \alpha_{t}-\alpha_{t}=0$. Combining this recursively with Inequality (40) implies in turn that $\alpha_{2}-\alpha_{1} \geq \alpha_{3}-\alpha_{2} \geq \cdots \geq \alpha_{t}-\alpha_{t-1} \geq 0$ so that the list of numbers $\left(\alpha_{1}, \ldots, \alpha_{k}\right)$ is increasing from 1 up to $t$. Similarly, applying Inequality (41) to $i^{\prime}=t$ implies that $\alpha_{t+1}-\alpha_{t} \leq \alpha_{t}-\alpha_{t}=0$. Combining this recursively with Inequality (41) satisfied for all $i^{\prime} \in\{t, \ldots, k-1\}$ (if any) leads to the conclusion that $\alpha_{k}-\alpha_{k-1} \leq \alpha_{k-1}-\alpha_{k-2} \leq \cdots \leq \alpha_{t+1}-\alpha_{t} \leq 0$ so that the list of numbers $\left(\alpha_{1}, \ldots, \alpha_{k}\right)$ is decreasing from $t$ up to $k$. Consider then any four integers $g, i, j$ and $l$ satisfying $1 \leq g<i \leq j<l \leq k$. Five cases need to be distinguished: 
(i) $g \geq t \geq 1$, then one has:

$$
\begin{aligned}
\alpha_{l}-\alpha_{j} & =\left(\alpha_{l}-\alpha_{l-1}\right)+\left(\alpha_{l-1}-\alpha_{l-2}\right)+\cdots+\left(\alpha_{j+1}-\alpha_{j}\right) \\
& \left.\leq \alpha_{j+1}-\alpha_{j} \text { (because the } \alpha_{h} \text { are decreasing above } t\right) \\
& \leq \alpha_{j}-\alpha_{t}(\text { by Inequality }(41)) \\
& =\alpha_{j}-\alpha_{i}+\alpha_{i}-\alpha_{g}+\alpha_{g}-\alpha_{t}(\text { for any integer } g, i, j) \\
& \leq \alpha_{i}-\alpha_{g}\left(\text { because the } \alpha_{h} \text { are decreasing above } t\right)
\end{aligned}
$$

(ii) $g<t \leq i \leq j<l \leq k$. Then one has:

$$
\begin{aligned}
\alpha_{l}-\alpha_{j} & =\left(\alpha_{l}-\alpha_{l-1}\right)+\left(\alpha_{l-1}-\alpha_{l-2}\right)+\cdots+\left(\alpha_{j+1}-\alpha_{j}\right) \\
& \left.\leq \alpha_{j+1}-\alpha_{j} \text { (because the } \alpha_{h} \text { are decreasing above } t\right) \\
& \leq \alpha_{j}-\alpha_{t}(\text { by Inequality }(41)) \\
& =\alpha_{j}-\alpha_{i}+\alpha_{i}-\alpha_{g}+\alpha_{g}-\alpha_{t}(\text { for any integer } g, i, j) \\
& \left.\leq \alpha_{i}-\alpha_{g} \text { (because } \alpha_{j}-\alpha_{i} \leq 0 \text { and } \alpha_{g}-\alpha_{t} \leq 0\right) .
\end{aligned}
$$

(iii) $g<i<t \leq j<l \leq k$. Then one has:

$$
\begin{aligned}
\alpha_{l}-\alpha_{j} & =\left(\alpha_{l}-\alpha_{l-1}\right)+\left(\alpha_{l-1}-\alpha_{l-2}\right)+\cdots+\left(\alpha_{j+1}-\alpha_{j}\right) \\
& \left.\leq \alpha_{j+1}-\alpha_{j} \text { (because the } \alpha_{h} \text { are decreasing above } t\right) \\
& \leq \alpha_{j}-\alpha_{t}(\text { by Inequality }(41)) \\
& \left.\leq 0 \text { (because the } \alpha_{h} \text { are decreasing above } t\right) \\
& \left.\leq \alpha_{i}-\alpha_{g} \text { (because the } \alpha_{h} \text { are increasing below } t\right) .
\end{aligned}
$$

(iv) $g<i \leq j<t \leq l \leq k$. Then one has:

$$
\begin{aligned}
\alpha_{i}-\alpha_{g} & =\left(\alpha_{i}-\alpha_{i-1}\right)+\left(\alpha_{i-1}-\alpha_{i-2}\right)+\cdots+\left(\alpha_{g+2}-\alpha_{g+1}\right)+\left(\alpha_{g+1}-\alpha_{g}\right) \\
& \left.\geq \alpha_{g+1}-\alpha_{g} \text { (because the } \alpha_{h} \text { are increasing below } t\right) \\
& \geq \alpha_{t}-\alpha_{g+1}(\text { by Inequality }(40)) \\
& =\alpha_{t}-\alpha_{l}+\alpha_{l}-\alpha_{j}+\alpha_{j}-\alpha_{g+1}(\text { for any } g+1 \leq j<l \leq k) \\
& \left.\geq \alpha_{l}-\alpha_{j} \text { (because } \alpha_{t}-\alpha_{l} \geq 0 \text { and } \alpha_{j}-\alpha_{g+1} \geq 0\right) .
\end{aligned}
$$

(v) $l<t \leq k$ In this case, one has:

$$
\begin{aligned}
\alpha_{i}-\alpha_{g} & =\left(\alpha_{i}-\alpha_{i-1}\right)+\left(\alpha_{i-1}-\alpha_{i-2}\right)+\cdots+\left(\alpha_{g+2}-\alpha_{g+1}\right)+\left(\alpha_{g+1}-\alpha_{g}\right) \\
& \left.\geq \alpha_{g+1}-\alpha_{g} \text { (because the } \alpha_{h} \text { are increasing below } t\right) \\
& \geq \alpha_{t}-\alpha_{g+1}(\text { by Inequality }(40)) \\
& \left.=\alpha_{t}-\alpha_{l}+\alpha_{l}-\alpha_{j}+\alpha_{j}-\alpha_{g+1} \text { (for any } g+1 \leq j<l \leq k\right) \\
& \left.\geq \alpha_{l}-\alpha_{j} \text { (because the } \alpha_{h} \text { are increasing below } t\right) .
\end{aligned}
$$


Hence, any list of $k$ numbers $\left(\alpha_{1}, \ldots, \alpha_{k}\right)$ or which there exists a $t \in\{1, \ldots, k\}$ such that Inequalities (41) and (40) belongs to $\mathcal{H}$.

\section{A.7 Theorem 3}

\section{A.7.1 Statement (a) implies Statement (b)}

Suppose $s$ has been obtained from $s^{\prime}$ by means of an increment. It then follows from Proposition 2 that Inequality (6) holds for all ordered lists of $k$ real numbers $\left(\alpha_{1}, \ldots, \alpha_{k}\right)$ in the set $\mathcal{A}_{F}$. This inequality holds therefore in particular for all such lists that belong to $\mathcal{A}_{H} \subset \mathcal{A}_{F}$. If, on the other hand, $s$ has been obtained from $s^{\prime}$ by means of a Hammond transfer, we know from Proposition 4 that Inequality (6) holds for all ordered lists of $k$ real numbers $\left(\alpha_{1}, \ldots, \alpha_{k}\right)$ in the set $\mathcal{H}$ and, therefore, for all ordered list of $k$ real numbers in the set $\mathcal{A}_{H} \subset \mathcal{H}$. The implication then follows from any finite repetition of these two elementary implications.

\section{A.7.2 Statement (b) implies Statement (c)}

Assume that Inequality (6) holds for all $\left(\alpha_{1}, \ldots, \alpha_{k}\right) \in \mathcal{A}_{\mathcal{H}}$. For any $i \in\{1, \ldots, k\}$, define the ordered list of $k$ numbers $\left(\alpha_{1}^{i}, \ldots, \alpha_{k}^{i}\right)$ by:

$$
\begin{aligned}
& \alpha_{h}^{i}=-\left(2^{i-h}\right), \text { for } h \in\{1, \ldots, i\} \\
& \alpha_{h}^{i}=0, \text { for } h \in\{i+1, \ldots, k\} .
\end{aligned}
$$

Let us first show that the ordered list $\left(\alpha_{1}^{i}, \ldots, \alpha_{k}^{i}\right)$ of real numbers thus defined belongs to $\mathcal{A}_{H}$ for every $i \in \mathcal{C}$. Thanks to Proposition 6 , this amounts to show that these real numbers satisfy:

$$
\alpha_{h+1}^{i}-\alpha_{h}^{i} \geq \alpha_{k}^{i}-\alpha_{h+1}^{i}
$$

for every $h \in\{1, \ldots, k-1\}$. If $h \geq i+1$, then one has:

$$
\alpha_{h+1}^{i}-\alpha_{h}^{i}=0-0=\alpha_{k}^{i}-\alpha_{h+1}^{i}
$$

so that Inequality (42) holds for that case. If $h=i$, then

$$
\alpha_{i+1}^{i}-\alpha_{i}^{i}=0+2^{0}>0-0=\alpha_{k}^{i}-\alpha_{i+1}^{i}
$$

so that (42) holds also for that case. If finally $h<i$, then one has:

$$
\begin{aligned}
\alpha_{h+1}^{i}-\alpha_{h}^{i} & =-2^{i-h-1}+2^{i-h} \\
& =2^{i-h-1} \\
& =0-\left(-2^{i-h-1}\right) \\
& =\alpha_{k}^{i}-\alpha_{h+1}^{i}
\end{aligned}
$$


so that (42) holds for this case as well. Since the ordered list $\left(\alpha_{1}^{i}, \ldots, \alpha_{k}^{i}\right)$ of real numbers belongs to $\mathcal{A}_{H}$ for every $i \in \mathcal{C}$, Inequality (6) must hold for any such ordered list of numbers. Hence, for every $i \in \mathcal{C}$, Inequality $\sum_{h=1}^{k} n_{h}^{s} \alpha_{h}^{i} \geq \sum_{h=1}^{k} n_{h}^{s^{\prime}} \alpha_{h}^{i}$ is equivalent to:

$$
\sum_{h=1}^{i}\left(2^{i-h}\right) n_{h}^{s} \leq \sum_{h=1}^{i}\left(2^{i-h}\right) n_{h}^{s^{\prime}}
$$

which is nothing else than the condition for $H$-dominance, as expressed by Eq. (9).

\section{A.7.3 Statement (c) implies Statement (a)}

Assume that $H(i ; s) \leq H\left(i ; s^{\prime}\right)$ for all $i \in\{1, \ldots, k-1\}$. We know from Proposition 1 that $F(i ; s) \leq F\left(i ; s^{\prime}\right)$ for all $i \in\{1, \ldots, k-1\}$ implies that $H(i ; s) \leq H\left(i ; s^{\prime}\right)$ for all $i \in\{1,2, \ldots, k-1\}$. If it is the case that, for all $i \in\{1, \ldots, k-1\}$, one has both $H(i ; s) \leq H\left(i ; s^{\prime}\right)$ and $F(i ; s) \leq F\left(i ; s^{\prime}\right)$, we conclude from Theorem 1 that $s$ can be obtained from $s^{\prime}$ by means of a finite sequence of increments and the proof is done. In the following, we therefore assume that $H(i ; s) \leq H\left(i ; s^{\prime}\right)$ holds for all $i \in\{1,2, \ldots, k-1\}$ but that there exists some $g \in\{1, \ldots, k-1\}$ for which one has $F(g ; s)-F\left(g ; s^{\prime}\right)>0$.

Step 1 Define then the index $h$ by:

$$
h=\min \left\{g \mid F(g ; s)-F\left(g ; s^{\prime}\right)>0\right\} .
$$

Given that index $h$, one can also define the index $l$ by:

$$
l=\min \left\{g>h \mid F(j ; s)-F\left(j ; s^{\prime}\right) \leq 0, \forall j \in\{g, g+1, \ldots, k\}\right\} .
$$

Such a $l$ exists because $F(k ; s)-F\left(k ; s^{\prime}\right)=0$. Notice that, by definition of $l$, one has:

$$
F(l-1 ; s)-F\left(l-1 ; s^{\prime}\right)>0 \text { and } F(l ; s)-F\left(l ; s^{\prime}\right) \leq 0,
$$

Hence, one has [using the definition of $F$ provided by (7)], that $n_{l}^{s}<n_{l}^{s^{\prime}}$. We now establish the existence of a unique $i \in\{1, \ldots, h-1\}$ such that:

$$
F(i ; s)-F\left(i ; s^{\prime}\right)<0 \text { and } F(g ; s)-F\left(g, s^{\prime}\right)=0, \forall g<i .
$$

Indeed, by assumption, we have $H(g ; s) \leq H\left(g ; s^{\prime}\right)$ for all $g=1,2, \ldots, k-1$. It follows that, thanks to Expression (10), Inequality $H(1 ; s) \leq H\left(1 ; s^{\prime}\right)$ is equivalent to:

$$
F(1 ; s)<F\left(1 ; s^{\prime}\right)
$$

or:

$$
F(1 ; s)=F\left(1 ; s^{\prime}\right) \text {. }
$$


If Case (47) holds, then the existence of some $i \in\{1, \ldots, h-1\}$ for which Expression (46) holds is established (in that case, $i=1$ ). Now assume that Case (48) holds. Since $H(2 ; s) \leq H\left(2 ; s^{\prime}\right)$ also holds, we must have $H(2 ; s)<H\left(2 ; s^{\prime}\right)$ or $H(2 ; s)=$ $H\left(2 ; s^{\prime}\right)$ which is, respectively, equivalent - thanks to Expression (11)—to:

$$
2 F(1 ; s)+F(2 ; s)<2 F\left(1 ; s^{\prime}\right)+F\left(2 ; s^{\prime}\right),
$$

or:

$$
2 F(1 ; s)+F(2 ; s)=2 F\left(1 ; s^{\prime}\right)+F\left(2 ; s^{\prime}\right)
$$

Again, if we are in Case (49), we can conclude [since $F(1 ; s)=F\left(1 ; s^{\prime}\right)$ ] that $F(2 ; s)<F\left(2 ; s^{\prime}\right)$, which establishes the existence of some $i \in\{1, \ldots, h-1\}$ for which Expression (46) holds (in that case, $i=2$ ). If we are in Case (50), we iterate in the same fashion using the definition of $H$ provided by (11). We notice that the index $i$ for which (46) holds must be strictly smaller than $h$, as defined in (43), because assuming otherwise will contradict the fact that $H(g ; s) \leq H\left(g ; s^{\prime}\right)$ holds for all $g \in\{1, \ldots, k-1\}$. We finally note that, because of the definition of $F$ provided by (7), the definition of the index $i$ just provided entails that:

$$
n_{i}^{s}<n_{i}^{s^{\prime}}, \quad \text { and } \quad n_{g}^{s}=n_{g}^{s^{\prime}}, \forall g \in\{1, \ldots, i-1\}
$$

Step 2 Given the indices $h$ and $l$ as defined in (43) and (44), respectively, we now proceed by defining a new society $-s^{1}$ say-obtained from $s^{\prime}$ by means of a Hammond transfer and such that $H(g ; s) \leq H\left(g ; s^{1}\right) \leq H\left(g ; s^{\prime}\right)$, for every $g \in\{1, \ldots, k-1\}$, with a strict inequality between $s^{1}$ and $s^{\prime}$ for at least one $g$. For this sake, we define the numbers $\delta_{1}, \delta_{2}$ and $\delta$ by:

$$
\delta_{1}=n\left[F\left(i ; s^{\prime}\right)-F(i ; s)\right], \quad \delta_{2}=n\left[F(l-1 ; s)-F\left(l-1 ; s^{\prime}\right)\right] \quad \text { and } \quad \delta=\min \left(\delta_{1}, \delta_{2}\right) .
$$

We note that, by the very definition of the index $i$, one has $\delta_{1}=n_{i}^{s^{\prime}}-n_{i}^{s}>0$. We notice also that, thanks to (45) and the definition of the index $l$, one has $0<\delta_{2} \leq n_{l}^{s^{\prime}}-n_{l}^{s}$. Define then the society $s^{1}$ by:

$$
\begin{gathered}
n_{g}^{s^{1}}=n_{g}^{s^{\prime}}, \forall g \neq i, i+1, l ; \\
n_{i}^{s^{1}}=n_{i}^{s^{\prime}}-\delta ; n_{i+1}^{s^{1}}=n_{i+1}^{s^{\prime}}+2 \delta ; n_{l}^{s^{1}}=n_{l}^{s^{\prime}}-\delta ;
\end{gathered}
$$

It is clear that $s^{1}$ has been obtained from $s^{\prime}$ by $\delta$ Hammond transfers as per Definition 3 where the indices $g, i, j$ and $l$ of this definition are, here, $i, i+1, i+1$ and $l$, respectively. After simple manipulation and the definition of $s^{1}$ in (53), we observe that: 


$$
F\left(g ; s^{1}\right)-F\left(g ; s^{\prime}\right)= \begin{cases}0 & \text { for } g \in\{1, \ldots, i-1\} \\ -\delta / n & \text { for } g=i \\ +\delta / n & \text { for } g \in\{i+1, \ldots, l-1\} \\ 0 & \text { for } g \in\{l, \ldots, k\}\end{cases}
$$

Again, after simple manipulation, one deduces that:

$$
H\left(g ; s^{1}\right)-H\left(g ; s^{\prime}\right)= \begin{cases}0 & \text { for } g \in\{1, \ldots, i-1\} \\ -\delta / n & \text { for } g=i \\ 0 & \text { for } g \in\{i+1, \ldots, l-1\} \\ -\left(2^{g-l}\right) \delta / n & \text { for } g \in\{l, \ldots, k\}\end{cases}
$$

Recalling that $\delta>0$ thanks to Expression (52), Expression (54) thus confirms that $H\left(g ; s^{1}\right) \leq H\left(g ; s^{\prime}\right)$, for every $g \in\{1, \ldots, k-1\}$, with strict inequalities for $g \in$ $i \cup\{l, \ldots, k\}$. Let us now verify that $H(g ; s) \leq H\left(g ; s^{1}\right)$, for all $g \in\{1, \ldots, k-1\}$. Because $\left[H(\cdot, s)-H\left(\cdot, s^{1}\right)\right]=\left[H(\cdot, s)-H\left(\cdot, s^{\prime}\right)\right]-\left[H\left(\cdot, s^{1}\right)-H\left(\cdot, s^{\prime}\right)\right]$, we first notice that Expression (54) entails that:

$$
H(g, s)-H\left(g, s^{1}\right)= \begin{cases}H(g, s)-H\left(g, s^{\prime}\right) & \text { for } g \in\{1, \ldots, i-1\}, \\ H(g, s)-H\left(g, s^{\prime}\right)+\delta / n & \text { for } g=i, \\ H(g, s)-H\left(g, s^{\prime}\right) & \text { for } g \in\{i+1, \ldots, l-1\} \\ H(g, s)-H\left(g, s^{\prime}\right)+\left(2^{g-l}\right) \delta / n & \text { for } g \in\{l, \ldots, k\}\end{cases}
$$

The fact that $H(g ; s)-H\left(g, s^{\prime}\right) \leq 0$ holds for all $g \in\{1, \ldots, k-1\}$ entails that $H(g ; s)-H\left(g ; s^{1}\right) \leq 0$ for all $g \in\{1,2, \ldots, i-1\} \cup\{i+1, \ldots, l-1\}$. Consider now the case $g=i$. Using (12), we know that:

$$
H(i ; s)-H\left(i, s^{\prime}\right)=2\left[H(i-1 ; s)-H\left(i-1 ; s^{\prime}\right)\right]+\left(n_{i}^{s}-n_{i}^{s^{\prime}}\right) / n
$$

By definition of $i$, one has $F(g ; s)-F\left(g ; s^{\prime}\right)=0$ for all $g<i$, so that the first term in the right-hand side of Eq. (56) is 0. Recalling then from (52) that $\delta_{1}=n_{i}^{s^{\prime}}-n_{i}^{s}>0$ and that $\delta=\min \left(\delta_{1}, \delta_{2}\right)$, it follows that:

$$
n_{i}^{s}-n_{i}^{s^{\prime}}+\delta \leq 0
$$

By combining Eqs. (55) and (56), we conclude that:

$$
H(i, s)-H\left(i, s^{1}\right)=H(i, s)-H\left(i, s^{\prime}\right)+\delta / n=\left(n_{i}^{s}-n_{i}^{s^{\prime}}+\delta\right) / n \leq 0 .
$$

Consider finally the case where $g \in\{l, \ldots, k-1\}$. By using Eq. (12), and recalling that $\delta_{2}=n\left[F(l-1 ; s)-F\left(l-1 ; s^{\prime}\right)\right]$, one has:

$H(l ; s)-H\left(l ; s^{\prime}\right)=2\left[H(l-1 ; s)-H\left(l-1 ; s^{\prime}\right)\right]+\left[F(l ; s)-F\left(l ; s^{\prime}\right)\right]-\delta_{2} / n$. 
Combining (58) with the last line of (55), and remembering that $\delta \leq \delta_{2}$, one obtains:

$$
\begin{aligned}
H(l, s)-H\left(l, s^{1}\right)= & 2\left[\left(H(l-1 ; s)-H\left(l-1 ; s^{\prime}\right)\right]+\left[F(l ; s)-F\left(l ; s^{\prime}\right)\right]\right. \\
& +\left(\delta-\delta_{2}\right) / n \leq 0 .
\end{aligned}
$$

Finally, using successive applications of Eq. (12), one obtains, for any $g \in\{l+$ $1, \ldots, k-1\}$ :

$$
\begin{aligned}
H(g ; s)-H\left(g ; s^{\prime}\right)= & \left(2^{g-l+1}\right)\left[H(l-1 ; s)-H\left(l-1 ; s^{\prime}\right)\right] \\
& +\sum_{j=l}^{g-1}\left(2^{g-j-1}\right)\left[F(j ; s)-F\left(j ; s^{\prime}\right)\right] \\
& +F(g ; s)-F\left(g ; s^{\prime}\right)-\left(2^{g-l}\right) \delta_{2} / n \\
\leq & 0
\end{aligned}
$$

since $F(j ; s) \leq F\left(j ; s^{\prime}\right)$ for all $j \in\{l, l+1, \ldots, k\}$. Combined with the last line of (55) and the fact that $\delta \leq \delta_{2}$, this completes the proof that $H(g ; s)-H\left(g ; s^{1}\right) \leq 0$ for all $g \in\{1, \ldots, k-1\}$. Hence, we have found a society $s^{1}$ obtained from society $s^{\prime}$ by means of a non-trivial Hammond transfers that is $H$-dominated by $s$.

Step 3 We now show that, in moving from $s^{\prime}$ to $s$, one has 'brought to naught' at least one of the differences $\left|F(h ; s)-F\left(h ; s^{\prime}\right)\right|$ that distinguishes $s$ from $s^{\prime}$. That is to say, we establish the existence of some $g \in\{1, \ldots, k-1\}$ for which one has $\left|F(g ; s)-F\left(g ; s^{1}\right)\right|=0$ and $\left|F(g ; s)-F\left(g ; s^{\prime}\right)\right|>0$. This is easily seen from the fact that, in the construction of $s^{1}$, one has either:

$$
\delta=\delta_{1}=n_{i}^{s^{\prime}}-n_{i}^{s}
$$

or:

$$
\delta=\delta_{2}=n\left[F(l-1 ; s)-F\left(l-1 ; s^{\prime}\right)\right] .
$$

If we are in Case (60), one has $F(i ; s)-F\left(i ; s^{1}\right)=0$ and $F(i ; s)-F\left(i ; s^{\prime}\right)<0$, by definition of the index $i$. If we are in Case $(61)$, then $F(l-1 ; s)-F\left(l-1 ; s^{1}\right)=$ $F(l-1, s)-F\left(l-1, s^{\prime}\right)+\delta_{2} / n=0$, and $F(l-1, s)-F\left(l-1, s^{\prime}\right)>0$, by definition of the index $l$.

Now, if $s=s^{1}$, then the proof is complete. If $s$ is distinct from $s^{1}$ but $s$ first-order dominates $s^{1}$, then we conclude that society $s$ can be obtained from society $s^{\prime}$ by means of a finite sequence of one Hammond transfer and a collection of increments (using Theorem 1). If $s$ is distinct from $s^{1}$ and $s$ does not first-order dominate $s^{1}$, then we can find three categories $i, h$ and $l$ just as in the preceding steps and construct a new distribution-say $s^{2}$ - that can be obtained from distribution $s^{1}$ by means of an (integer number of) Hammond transfers and that is $H$-dominated by $s$ and so on. More generally, after a finite number — $t$ say-of iterations, we will find a distribution $s^{t}$ obtained from $s^{\prime}$ by means of $t$ Hammond transfers such that $s H$-dominates $s^{t}$. In that case, we will have either $s=s^{t}$ or $s$ first-order dominates $s^{t}$. Since there 
are finitely many differences of the kind $\left|F(g ; s)-F\left(g ; s^{\prime}\right)\right|$ to bring to naught, the number $t$ must be finite. This completes the proof.

\section{A.8 Lemma 2}

Step 1 Using (19) in Lemma 1, we have, for any society $s$ :

$$
\frac{1}{n} \sum_{h=1}^{k} n_{h}^{s} \alpha_{h}=\alpha_{k}-\sum_{h=1}^{k-1} F(h ; s)\left[\alpha_{h+1}-\alpha_{h}\right] .
$$

In order to simplify the notation we let, in this proof, $F_{h}=F(h ; s)$ and $\theta_{h}=\left(\alpha_{h+1}-\right.$ $\alpha_{h}$ ) so that:

$$
\frac{1}{n} \sum_{i=1}^{k} n_{h}^{s} \alpha_{h}=\alpha_{k}-\sum_{h=1}^{k-1} F_{h} \theta_{h}
$$

Letting $\vartheta_{h}=\theta_{h}-\sum_{j=h+1}^{k-1} \theta_{j}$ for all $h \in\{1, \ldots, k-2\}$ and $\vartheta_{k-1}=\theta_{k-1}$, we rewrite each term of the sum $\sum_{h=1}^{k-1} F_{h} \theta_{h}$ in (63) as follows:

For $h=1$ :

$$
\begin{aligned}
F_{1} \theta_{1} & =F_{1}\left[\theta_{1}-\sum_{j=2}^{k-1} \theta_{j}\right]+F_{1} \theta_{2}+F_{1} \theta_{3}+\cdots+F_{1} \theta_{k-1} \\
& =F_{1}\left[\theta_{1}-\sum_{j=2}^{k-1} \theta_{j}\right]+F_{1}\left[\theta_{2}-\sum_{j=3}^{k-1} \theta_{j}\right]+2 F_{1} \theta_{3}+\cdots+2 F_{1} \theta_{k-1} \\
& =F_{1}\left[\theta_{1}-\sum_{j=2}^{k-1} \theta_{j}\right]+F_{1}\left[\theta_{2}-\sum_{j=3}^{k-1} \theta_{j}\right]+2 F_{1}\left[\theta_{3}-\sum_{j=4}^{k-1} \theta_{j}\right]+\cdots+4 F_{1} \theta_{k-1} \\
& =\cdots \\
& =F_{1} \vartheta_{1}+F_{1} \vartheta_{2}+2 F_{1} \vartheta_{3}+\left(2^{2}\right) F_{1} \vartheta_{4}+\cdots+\left(2^{k-3}\right) F_{1} \vartheta_{k-1} .
\end{aligned}
$$

For $h=2:$

$$
\begin{aligned}
F_{2} \theta_{2} & =F_{2}\left[\theta_{2}-\sum_{j=3}^{k-1} \theta_{j}\right]+F_{2} \theta_{3}+F_{2} \theta_{4}+\cdots+F_{2} \theta_{k-1} \\
& =F_{2}\left[\theta_{2}-\sum_{j=3}^{k-1} \theta_{j}\right]+F_{2}\left[\theta_{3}-\sum_{j=4}^{k-1} \theta_{j}\right]+2 F_{2} \theta_{4}+\cdots+2 F_{2} \theta_{k-1} \\
& =F_{2}\left[\theta_{2}-\sum_{j=3}^{k-1} \theta_{j}\right]+F_{2}\left[\theta_{3}-\sum_{j=4}^{k-1} \theta_{j}\right]+2 F_{2}\left[\theta_{4}-\sum_{j=5}^{k-1} \theta_{j}\right]+\cdots+4 F_{2} \theta_{k-1}
\end{aligned}
$$




$$
\begin{aligned}
& =\cdots \\
& =F_{2} \vartheta_{2}+F_{2} \vartheta_{3}+2 F_{2} \vartheta_{4}+\left(2^{2}\right) F_{2} \vartheta_{5}+\cdots+\left(2^{k-4}\right) F_{2} \vartheta_{k-1} .
\end{aligned}
$$

More generally, we have $F_{k-1} \theta_{k-1}=F_{k-1} \vartheta_{k-1}$ and:

$$
F_{h} \theta_{h}=F_{h} \vartheta_{h}+F_{h} \sum_{j=i+1}^{k-1}\left(2^{j-i-1}\right) \vartheta_{j}, \quad \forall i \in\{1, \ldots, k-2\} .
$$

Hence, one can write:

$$
\begin{aligned}
& F_{1} \theta_{1}=F_{1} \vartheta_{1}+F_{1} \vartheta_{2}+2 F_{1} \vartheta_{3}+\left(2^{2}\right) F_{1} \vartheta_{4}+\cdots+\left(2^{k-4}\right) F_{1} \vartheta_{k-2}+\left(2^{k-3}\right) F_{1} \vartheta_{k-1} \\
& F_{2} \theta_{2}=\quad F_{2} \vartheta_{2}+F_{2} \vartheta_{3}+2 F_{2} \vartheta_{4}+\cdots+\left(2^{k-5}\right) F_{2} \vartheta_{k-2}+\left(2^{k-4}\right) F_{2} \vartheta_{k-1} \\
& F_{3} \theta_{3}=\quad F_{3} \vartheta_{3}+F_{3} \vartheta_{4}+\cdots+\left(2^{k-6}\right) F_{3} \vartheta_{k-2}+\left(2^{k-5}\right) F_{3} \vartheta_{k-1} \\
& F_{4} \theta_{4}=\quad+F_{4} \vartheta_{4}+\cdots+\left(2^{k-7}\right) F_{4} \vartheta_{k-2}+\left(2^{k-6}\right) F_{4} \vartheta_{k-1} \\
& F_{k-2} \theta_{k-2}=\quad F_{k-2} \vartheta_{k-2}+F_{k-2} \vartheta_{k-1} \\
& F_{k-1} \theta_{k-1}=
\end{aligned}
$$

Remembering that $\vartheta_{k-1}=\theta_{k-1}$ and $\vartheta_{h}=\theta_{h}-\sum_{j=h+1}^{k-1} \theta_{j}$ for all $h \in\{1, \ldots, k-2\}$, one can use Eq. (11) and sum vertically the decomposition (67) to obtain:

$$
\sum_{h=1}^{k-1} F_{h} \theta_{h}=\sum_{h=1}^{k-2} H(h ; s)\left[\theta_{h}-\sum_{j=h+1}^{k-1} \theta_{j}\right]+H(k-1 ; s) \theta_{k-1} .
$$

Since $\frac{1}{n} \sum_{h=1}^{k} n_{h}^{s} \alpha_{h}=\alpha_{k}-\sum_{h=1}^{k-1} F_{h} \theta_{h}$, one obtains finally:

$$
\frac{1}{n} \sum_{h=1}^{k} n_{h}^{s} \alpha_{h}=\alpha_{k}-\sum_{h=1}^{k-2} H(h ; s)\left[\theta_{h}-\sum_{j=h+1}^{k-1} \theta_{j}\right]-H(k-1 ; s) \theta_{k-1} \text {. }
$$

Step 2. In a symmetric fashion, one obtains from Eq. (20) in Lemma 1:

$$
\frac{1}{n} \sum_{h=1}^{k} n_{h}^{s} \alpha_{h}=\alpha_{1}+\sum_{h=1}^{k-1} \bar{F}(h ; s)\left[\alpha_{h+1}-\alpha_{h}\right]
$$

Letting $\bar{F}_{h}=\bar{F}(h ; s)$ and $\theta_{h}=\left(\alpha_{h+1}-\alpha_{h}\right)$, we have:

$$
\frac{1}{n} \sum_{h=1}^{k} n_{h}^{s} \alpha_{h}=\alpha_{1}+\sum_{h=1}^{k-1} \bar{F}_{h} \theta_{h}
$$

Now, letting $\beta_{1}=\theta_{1}$ and $\beta_{h}=\left(\theta_{h}-\sum_{j=1}^{h-1} \theta_{j}\right)$ for all $h \in\{2, \ldots, k-1\}$, we propose to rewrite $\sum_{h=1}^{k-1} \bar{F}_{h} \theta_{h}$ in (71) as follows: 
$\underline{\text { For } h=k-1:}$

$$
\begin{aligned}
& \bar{F}_{k-1} \theta_{k-1}=\bar{F}_{k-1}\left[\theta_{k-1}-\sum_{j=1}^{k-2} \theta_{j}\right]+\bar{F}_{k-1} \theta_{k-2}+\bar{F}_{k-1} \theta_{k-3}+\cdots+\bar{F}_{k-1} \theta_{1} \\
& =\bar{F}_{k-1}\left[\theta_{k-1}-\sum_{j=1}^{k-2} \theta_{j}\right]+\bar{F}_{k-1}\left[\theta_{k-2}-\sum_{j=1}^{k-3} \theta_{j}\right] \\
& +2 \bar{F}_{k-1} \theta_{k-3}+\cdots+2 \bar{F}_{k-1} \theta_{1} \\
& =\bar{F}_{k-1}\left[\theta_{k-1}-\sum_{j=1}^{k-2} \theta_{j}\right]+\bar{F}_{k-1}\left[\theta_{k-2}-\sum_{j=1}^{k-3} \theta_{j}\right] \\
& +2 \bar{F}_{k-1}\left[\theta_{k-3}-\sum_{j=1}^{k-4} \theta_{j}\right]+\cdots+4 \bar{F}_{k-1} \theta_{1} \\
& =\cdots \\
& =\bar{F}_{k-1} \beta_{k-1}+\bar{F}_{k-1} \beta_{k-2}+2 \bar{F}_{k-1} \beta_{k-3} \\
& +\left(2^{2}\right) \bar{F}_{k-1} \beta_{k-4}+\cdots+\left(2^{k-3}\right) \bar{F}_{k-1} \beta_{1} \text {. }
\end{aligned}
$$

For $h=k-2$ :

$$
\begin{aligned}
& \bar{F}_{k-2} \theta_{k-2}=\bar{F}_{k-2}\left[\theta_{k-2}-\sum_{j=1}^{k-3} \theta_{j}\right]+\bar{F}_{k-2} \theta_{k-3}+\bar{F}_{k-2} \theta_{k-4}+\cdots+\bar{F}_{k-2} \theta_{1} \\
& =\bar{F}_{k-2}\left[\theta_{k-2}-\sum_{j=1}^{k-3} \theta_{j}\right]+\bar{F}_{k-2}\left[\theta_{k-3}-\sum_{j=1}^{k-4} \theta_{j}\right] \\
& +2 \bar{F}_{k-2} \theta_{k-4}+\cdots+2 \bar{F}_{k-2} \theta_{1} \\
& =\bar{F}_{k-2}\left[\theta_{k-2}-\sum_{j=1}^{k-3} \theta_{j}\right]+\bar{F}_{k-2}\left[\theta_{k-3}-\sum_{j=1}^{k-4} \theta_{j}\right] \\
& +2 \bar{F}_{k-2}\left[\theta_{k-4}-\sum_{j=1}^{k-5} \theta_{j}\right]+\cdots+4 \bar{F}_{k-2} \theta_{1} \\
& =\cdots \\
& =\bar{F}_{k-2} \beta_{k-2}+\bar{F}_{k-2} \beta_{k-3}+2 \bar{F}_{k-2} \beta_{k-4} \\
& +\left(2^{2}\right) \bar{F}_{k-2} \beta_{k-5}+\cdots+\left(2^{k-4}\right) \bar{F}_{k-2} \beta_{1} \text {. }
\end{aligned}
$$

More generally, one has $\bar{F}_{1} \theta_{1}=\bar{F}_{1} \beta_{1}$ and:

$$
\bar{F}_{h} \theta_{h}=\bar{F}_{h} \beta_{h}+\bar{F}_{h} \sum_{j=1}^{h-1}\left(2^{h-j-1}\right) \beta_{j}, \quad \forall h \in\{2, \ldots, k-1\} .
$$


Hence, one can conclude that:

$$
\begin{aligned}
& \bar{F}_{k-1} \theta_{k-1}=\bar{F}_{k-1} \beta_{k-1}+\bar{F}_{k-1} \beta_{k-2}+2 \bar{F}_{k-1} \beta_{k-3}+\left(2^{2}\right) \bar{F}_{k-1} \beta_{k-4}+\cdots+\left(2^{k-4}\right) \bar{F}_{k-1} \beta_{2}+\left(2^{k-3}\right) \bar{F}_{k-1} \beta_{1} \\
& \bar{F}_{k-2} \theta_{k-2}=\quad \bar{F}_{k-2} \beta_{k-2}+\bar{F}_{k-2} \beta_{k-3}+2 \bar{F}_{k-2} \beta_{k-4}+\cdots+\left(2^{k-5}\right) \bar{F}_{k-2} \beta_{2}+\left(2^{k-4}\right) \bar{F}_{k-2} \beta_{1} \\
& \bar{F}_{k-3} \theta_{k-3}=\quad \bar{F}_{k-3} \beta_{k-3}+\bar{F}_{k-3} \beta_{k-4}+\cdots+\left(2^{k-6}\right) \bar{F}_{k-3} \beta_{2}+\left(2^{k-5}\right) \bar{F}_{k-3} \beta_{1} \\
& \bar{F}_{k-4} \theta_{k-4}=\quad+\bar{F}_{k-4} \beta_{k-4}+\cdots+\left(2^{k-7}\right) \bar{F}_{k-4} \beta_{2}+\left(2^{k-6}\right) \bar{F}_{k-4} \beta_{1} \\
& \stackrel{\bar{F}_{2} \theta_{2}=}{\bar{F}_{1}} \bar{F}_{2} \beta_{2}+\bar{F}_{2} \beta_{1} \\
& \bar{F}_{1} \theta_{1}=
\end{aligned}
$$

Using (16), and summing vertically the previous equation, one obtains:

$$
\sum_{h=1}^{k-1} \bar{F}_{h} \theta_{h}=\bar{H}(1 ; s) \theta_{1}+\sum_{h=2}^{k-1} \bar{H}(h ; s)\left[\theta_{h}-\sum_{j=1}^{h-1} \theta_{j}\right] .
$$

Since $\frac{1}{n} \sum_{h=1}^{k} n_{h}^{s} \alpha_{h}=\alpha_{1}+\sum_{h=1}^{k-1} \bar{F}_{h} \theta_{h}$, we finally obtain:

$$
\frac{1}{n} \sum_{h=1}^{k} n_{h}^{s} \alpha_{h}=\alpha_{1}+\bar{H}(1 ; s) \theta_{1}+\sum_{h=2}^{k-1} \bar{H}(h ; s)\left[\theta_{h}-\sum_{j=1}^{h-1} \theta_{j}\right] .
$$

STEP 3. From Eq. (21) in Lemma 1, one has, for any $t \in\{2, \ldots, k-1\}$ :

$$
\frac{1}{n} \sum_{h=1}^{k} n_{h}^{s} \alpha_{h}=\alpha_{t}-\sum_{h=1}^{t-1} F_{h} \theta_{h}+\sum_{h=t}^{k-1} \bar{F}_{h} \theta_{h} .
$$

By using Eq. (68) and replacing category $k$ by category $t$ in this equation, we obtain:

$$
\sum_{h=1}^{t-1} F_{h} \theta_{h}=\sum_{h=1}^{t-2} H(h ; s)\left[\theta_{h}-\sum_{j=h+1}^{t-1} \theta_{j}\right]+H(t-1 ; s) \theta_{t-1} .
$$

Symmetrically, replacing category 1 by category $t$ in Eq. (76) enables one to write::

$$
\sum_{h=t}^{k-1} \bar{F}_{h} \theta_{h}=\bar{H}(t ; s) \theta_{t}+\sum_{h=t+1}^{k-1} \bar{H}(h ; s)\left[\theta_{h}-\sum_{j=t}^{h-1} \theta_{j}\right] .
$$

Combining Eqs. (78), (79) and (80), one gets finally the desired result.

\section{A.9 Theorem 5}

\section{A.9.1 Statement (a) implies Statement (b)}

That results immediately from the definition of the set $\mathcal{H}$ (using Proposition 4). 


\section{A.9.2 Statement (b) implies Statement (a)}

The proof builds on the dual theory for convex cones, recently investigated by Muller and Scarsini (2012), but adapted to our fully discrete framework. An more detailed description of the proof-stated here for completeness - can be found in Magdalou (2018). (See in particular the discussion on p. 17.) First, we define by $\mathcal{E}$ the set of all lists $\alpha=\left(\alpha_{1}, \ldots, \alpha_{k}\right) \in \mathbb{R}^{k}$ which assign numerical evaluations $\alpha_{h}$ to each category $h \in \mathcal{C}$, and such that there exist $i, j \in \mathcal{C}$ with $\alpha_{i} \neq \alpha_{j}$. We then denote by $\mathcal{M}$ the set of all lists $m=\left(m_{1}, \ldots, m_{k}\right) \in \mathbb{R}^{k}$ such that $\sum_{h=1}^{k} m_{h}=0$. For all $m \in \mathcal{M}$ and all $\alpha \in \mathcal{E}$, we define the bilinear mapping $b(\cdot, \cdot)$ by $b(m, \alpha)=-\sum_{h=1}^{k} m_{h} \alpha_{h}$. The pair $(\mathcal{M}, \mathcal{E})$ is thus, under the bilinear mapping $b(\cdot, \cdot)$, a dual pair, what we denote $(\mathcal{M}, \mathcal{E} ; b)$. The dual pair $(\mathcal{M}, \mathcal{E} ; b)$ is moreover strict and is the sense that for each $0 \neq m \in \mathcal{M}$, there is an $\alpha \in \mathcal{E}$ with $b(m, \alpha) \neq 0$ and, for each $0 \neq \alpha \in \mathcal{E}$, there is a $m \in \mathcal{M}$ with $b(m, \alpha) \neq 0$.

We then recall that any two societies $s$ and $s^{\prime}$ are characterized by the lists $n^{s}=$ $\left(n_{1}^{s}, \ldots, n_{k}^{s}\right)$ and $n^{s^{\prime}}=\left(n_{1}^{s^{\prime}}, \ldots, n_{k}^{s^{\prime}}\right)$, respectively, which indicate the number of agents in each category in $\mathcal{C}$, and such that $\sum_{h=1}^{k} n_{h}^{s}=\sum_{h=1}^{k} n_{h}^{s^{\prime}}=n$. We define by $\mathcal{T} \subset \mathcal{M}$ the set of all lists $\left(n^{s}-n^{s^{\prime}}\right)$ such that $s$ can be obtained from $s^{\prime}$ by means of only one Hammond transfer.

Consider now two particular societies $s$ and $s^{\prime}$ and assume that Statement (b) is true, so that inequality $\sum_{h=1}^{k} n_{h}^{s} \alpha_{h} \geq \sum_{h=1}^{k} n_{h}^{s^{\prime}} \alpha_{h}$, or equivalently $b\left(n^{s}-n^{s^{\prime}}, \alpha\right) \leq 0$, holds for all lists $\alpha \in \mathcal{H}$. We have to establish that $s$ can be obtained from $s^{\prime}$ by means of a finite sequence of Hammond transfers. Consider first the set of all constant lists $\alpha \in \mathcal{H}$, such that $\alpha_{i+1}=\alpha_{i}$ for all $i \in\{1, \ldots, k-1\}$. The only information provided by this subclass of $\mathcal{H}$ is that $\sum_{h=1}^{k} n_{h}^{s}=\sum_{h=1}^{k} n_{h}^{s^{\prime}}$, which is actually known by assumption. Hence, we restrict attention to the set $\tilde{\mathcal{H}}$ of all lists $\alpha \in \mathcal{H}$, such that there exist $i, j \in \mathcal{C}$ with $\alpha_{i} \neq \alpha_{j}$.

The polar cone of $\tilde{\mathcal{H}} \subset \mathcal{E}$ under the duality $(\mathcal{M}, \mathcal{E} ; b)$, which is denoted by $\tilde{\mathcal{H}}^{\circ}$, is defined by:

$$
\tilde{\mathcal{H}}^{\circ}=\{m \in \mathcal{M} \mid b(m, \alpha) \leq 0, \forall \alpha \in \tilde{\mathcal{H}}\}
$$

By definition, $\left(n^{s}-n^{s^{\prime}}\right) \in \mathcal{M}$. Because we have assumed that $b\left(n^{s}-n^{s^{\prime}}, \alpha\right) \leq 0$ holds for all $\alpha \in \mathcal{H}$, this also holds for all $\alpha \in \tilde{\mathcal{H}}$ (as $\tilde{\mathcal{H}} \subset \mathcal{H}$ ). Hence, we also have $\left(n^{s}-n^{s^{\prime}}\right) \in \tilde{\mathcal{H}}^{\circ}$. Now, the polar cone of the set $\mathcal{T} \subset \mathcal{M}$ under the duality $(\mathcal{M}, \mathcal{E} ; b)$, which is denoted by $\mathcal{T}^{\circ}$, is defined by:

$$
\mathcal{T}^{\circ}=\{\alpha \in \mathcal{E} \mid b(m, \alpha) \leq 0, \forall m \in \mathcal{T}\}
$$

Thanks to Proposition 4, one immediately deduces that $\tilde{\mathcal{H}}=\mathcal{T}^{\circ}$. Because $\left(n^{s}-n^{s^{\prime}}\right) \in$ $\tilde{\mathcal{H}}^{\circ}$ and $\tilde{\mathcal{H}}=\mathcal{T}^{\circ}$, one concludes that $\left(n^{s}-n^{s^{\prime}}\right) \in \mathcal{T}^{\circ \circ}$, where $\mathcal{T}^{\circ \circ}$ is the bipolar cone of $\mathcal{T}$.

We then let $\mathcal{D}_{o}(\mathcal{T})=\operatorname{co}\left\{\lambda m \mid \lambda \in \mathbb{R}_{+}, m \in \mathcal{T}\right\}$, where co indicates the convex hull of the set. As $\mathcal{T}$ is a discrete and finite set, $\mathcal{D}_{o}(\mathcal{T})$ is closed. By applying the bipolar 
theorem, one deduces that $\mathcal{T}^{\circ \circ}=\mathcal{D}_{o}(\mathcal{T})$. One also remarks that, by definition, $\mathcal{T} \subset \mathbb{Z}^{k}$ where $\mathbb{Z}$ is the set of integers. Thus, $\left(n^{s}-n^{s^{\prime}}\right) \in \mathcal{D}_{o}(\mathcal{T}) \cap \mathbb{Z}^{k}$. By applying the notion of minimal Hilbert basis [see Magdalou (2018)], it can be shown that, actually, there exists a positive and finite integer $t$ such that $\left(n^{s}-n^{s^{\prime}}\right)=\sum_{h=1}^{t} \lambda_{h} m_{h}$, where $m_{h} \in \mathcal{T}$ and $\lambda_{h} \in \mathbb{Z}_{+}$-with $\mathbb{Z}_{+}$the nonnegative orthant of $\mathbb{Z}$ - for all $h \in\{1, \ldots, t\}$. That concludes the proof.

\section{A.9.3 Statement (b) implies Statement (c)}

Assume that the inequality $\sum_{h=1}^{k} n_{h}^{s} \alpha_{h} \geq \sum_{h=1}^{k} n_{h}^{s^{\prime}} \alpha_{h}$ holds for all lists $\left(\alpha_{1}, \ldots, \alpha_{k}\right) \in$ $\mathcal{H}$. This implies in particular that the inequality holds for all $\left(\alpha_{1}, \ldots, \alpha_{k}\right) \in \mathcal{A}_{H}$ (as $\mathcal{A}_{H} \subset \mathcal{H}$ ). It then follows from Theorem 3 that society $s H$-dominates society $s^{\prime}$. Similarly, the fact that the inequality $\sum_{h=1}^{k} n_{h}^{s} \alpha_{h} \geq \sum_{h=1}^{k} n_{h}^{s^{\prime}} \alpha_{h}$ holds for all lists $\left(\alpha_{1}, \ldots, \alpha_{k}\right) \in \mathcal{H}$ implies in particular that it holds for all $\left(\alpha_{1}, \ldots, \alpha_{k}\right) \in \mathcal{A}_{\bar{H}}$ (as $\left.\mathcal{A}_{\bar{H}} \subset \mathcal{H}\right)$. Hence, thanks to Theorem 4 , society $s \bar{H}$-dominates society $s^{\prime}$. We conclude that society $s$ both $H$-dominates and $\bar{H}$-dominates society $s^{\prime}$.

\section{A.9.4 Statement (c) implies Statement (b)}

Assume that $H(h, s) \leq H\left(h, s^{\prime}\right)$ and $\bar{H}(h, s) \leq \bar{H}\left(h, s^{\prime}\right)$ for every category $h \in\{1, \ldots, k-1\}$. Thanks to Proposition 5, we have to show that $\sum_{h=1}^{k} n_{h}^{s} \alpha_{h} \geq$ $\sum_{h=1}^{k} n_{h}^{s^{\prime}} \alpha_{h}$ must hold as well for $\left(\alpha_{1}, \ldots, \alpha_{k}\right) \in \mathbb{R}^{k}$ for which there exists an integer $t \in\{1, \ldots, k\}$ such that $\left(\alpha_{i+1}-\alpha_{i}\right) \geq\left(\alpha_{t}-\alpha_{i+1}\right)$, for all $i \in\{1, \ldots, t-1\}$ (if any) and $\left(\alpha_{i^{\prime}+1}-\alpha_{i^{\prime}}\right) \leq\left(\alpha_{i^{\prime}}-\alpha_{t}\right)$, for all $i^{\prime} \in\{t, \ldots, k-1\}$ (again if this set is non-empty).

Consider that such a $t$ exists. If $t=k$, we know from Proposition 6 that $\left(\alpha_{1}, \ldots, \alpha_{k}\right) \in \mathcal{A}_{H}$. Because by assumption $H(h, s) \leq H\left(h, s^{\prime}\right)$ for every category $h \in\{1, \ldots, k-1\}$ we know, thanks to Theorem 3, that Inequality $\sum_{h=1}^{k} n_{h}^{s} \alpha_{h} \geq$ $\sum_{h=1}^{k} n_{h}^{s^{\prime}} \alpha_{h}$ holds. Symmetrically, if $t=1$, it follows from Proposition 7 that $\left(\alpha_{1}, \ldots, \alpha_{k}\right) \in \mathcal{A}_{\bar{H}}$. Because by assumption $\bar{H}(h, s) \leq \bar{H}\left(h, s^{\prime}\right)$ for every category $h \in\{1, \ldots, k-1\}$ we know, thanks to Theorem 4 , that Inequality $\sum_{h=1}^{k} n_{h}^{s} \alpha_{h} \geq$ $\sum_{h=1}^{k} n_{h}^{s^{\prime}} \alpha_{h}$ holds again. Finally, consider that $t \in\{2, \ldots, k-1\}$. By definition of $t$, we have:

$$
\begin{gathered}
\theta_{t-1} \geq 0, \quad \text { and }\left[\theta_{h}-\sum_{j=h+1}^{t-1} \theta_{j}\right] \geq 0, \forall h \in\{1, \ldots, t-2\} ; \\
\theta_{t} \leq 0, \quad \text { and }\left[\theta_{h}-\sum_{j=t}^{h-1} \theta_{j}\right] \leq 0, \forall h \in\{t+1, \ldots, k-1\} .
\end{gathered}
$$

Letting $\theta_{h}=\left(\alpha_{h+1}-\alpha_{h}\right)$ for every $h \in\{1, \ldots, k-1\}$ we know, thanks to the decomposition result of Lemma 2, that: 


$$
\begin{aligned}
\frac{1}{n}\left[\sum_{h=1}^{k} n_{h}^{s} \alpha_{h}-\sum_{h=1}^{k} n_{h}^{s^{\prime}} \alpha_{h}\right] & =\sum_{h=1}^{t-2}\left[H\left(h ; s^{\prime}\right)-H(h ; s)\right]\left[\theta_{h}-\sum_{j=h+1}^{t-1} \theta_{j}\right] \\
& +\left[H\left(t-1 ; s^{\prime}\right)-H(t-1 ; s)\right] \theta_{t-1} \\
& +\left[\bar{H}(t ; s)-\bar{H}\left(t ; s^{\prime}\right)\right] \theta_{t} \\
& +\sum_{h=t+1}^{k-1}\left[\bar{H}(h ; s)-\bar{H}\left(h ; s^{\prime}\right)\right]\left[\theta_{h}-\sum_{j=t}^{h-1} \theta_{j}\right] .
\end{aligned}
$$

Since by assumption $H(h, s) \leq H\left(h, s^{\prime}\right)$ and $\bar{H}(h, s) \leq \bar{H}\left(h, s^{\prime}\right)$ for every category $h \in\{1, \ldots, k-1\}$, combining this information with Eqs. (83) and (84) leads to the required conclusion that Expression (85) is nonnegative.

\section{A.10 Proposition 8}

Assume that $s$ and $s^{\prime}$ are two distinct societies for which $H(h, s) \leq H\left(h, s^{\prime}\right)$ holds for all categories $h \in\{1, \ldots, k-1\}$. It follows from the recursive definition of the $H$-curve provided by Eqs. (10) and (12) that the smallest $i \in\{1,2, \ldots, k\}$ for which $n_{i}^{s} \neq n_{i}^{s^{\prime}}$ is such that $n_{i}^{s}<n_{i}^{s^{\prime}}$. But this implies that $s \succeq_{L} s^{\prime}$.

\section{A.11 Proposition 9}

$\underline{\text { STEP } 1}$. As a preliminary of the proof, we first notice that, for any society $s$ defined on the grid $\mathcal{C}(t)$, one has, for any $t \in\{0,1, \ldots\}$ :

$$
n^{s}\left(\frac{2 i+1}{2^{t+1}}\right)=0,
$$

and:

$$
H^{t+1}\left(\frac{2 i+1}{2^{t+1}} ; s\right)=2 H^{t+1}\left(\frac{i}{2^{t}} ; s\right) .
$$

Indeed, we notice that:

$$
\mathcal{C}(t)=\left\{\frac{1}{2^{t}}, \frac{2}{2^{t}}, \ldots, \frac{\left(2^{t}\right) k}{2^{t}}\right\}, \quad \text { and } \mathcal{C}(t+1)=\left\{\frac{1}{2^{t+1}}, \frac{2}{2^{t+1}}, \ldots, \frac{\left(2^{t+1}\right) k}{2^{t+1}}\right\},
$$

and that:

$$
\frac{i}{2^{t}}=\frac{2 i}{2^{t+1}}, \quad \forall \in\left\{1,2, \ldots,\left(2^{t}\right) k\right\}
$$


Equation (86) then follows from the fact that $n^{s}(x)=0$ for all $x \notin \mathcal{C}(t)$, while Eq. (87) is an immediate consequence of Eqs. (86) and (89) and the fact that, thanks to Expression (12), one has:

$H^{t+1}\left(\frac{2 i+1}{2^{t+1}} ; s\right)=2 H^{t+1}\left(\frac{2 i}{2^{t+1}} ; s\right)+n^{s}\left(\frac{2 i+1}{2^{t+1}}\right) / n, \quad \forall i \in\left\{0,1, \ldots,\left(2^{t}\right) k-1\right\}$.

STEP 2. We now observe that, for any society $s$ :

$$
H^{t+1}\left(\frac{i}{2^{t}} ; s\right)=\sum_{h=1}^{i-1}\left(2^{2(i-h)-1}\right) H^{t}\left(\frac{h}{2^{t}} ; s\right)+H^{t}\left(\frac{i}{2^{t}} ; s\right), \quad \forall i \in\left\{0,1, \ldots,\left(2^{t}\right) k\right\}
$$

Indeed, from Eq. (26) applied to the grid $\mathcal{C}(t+1)$, we know that:

$$
H^{t+1}(x ; s)=\frac{1}{n} \sum_{h=1}^{j}\left(2^{j-h}\right) n^{s}\left(\frac{h}{2^{t+1}}\right)
$$

for any $x \in \mathcal{C}(t+1)$, and $j=x 2^{t+1}$. Applying this to $x=i / 2^{t}$ for any $i \in$ $\left\{1, \ldots,\left(2^{t}\right) k\right\}$ yields:

$$
H^{t+1}\left(\frac{i}{2^{t}} ; s\right)=\frac{1}{n} \sum_{h=1}^{2 i}\left(2^{2 i-h}\right) n^{s}\left(\frac{h}{2^{t+1}}\right) .
$$

Expression (90) can then be obtained from (91) and the following observations (made only for $i=1,2,3$, but easily extendable to any other $i$ ). For $i=1,2,3$, and recalling that $n^{s}\left(\frac{h}{2^{t+1}}\right)=0$ as soon as $h$ is uneven, Expression (91) writes indeed as:

$$
\begin{aligned}
H^{t+1}\left(\frac{1}{2^{t}} ; s\right) & =\frac{1}{n}\left[2 n^{s}\left(\frac{1}{2^{t+1}}\right)+n^{s}\left(\frac{2}{2^{t+1}}\right)\right] \\
& =\frac{1}{n} n^{s}\left(\frac{2}{2^{t+1}}\right) \\
& =\frac{1}{n} n^{s}\left(\frac{1}{2^{t}}\right), \\
H^{t+1}\left(\frac{2}{2^{t}} ; s\right) & =\frac{1}{n}\left[8 n^{s}\left(\frac{1}{2^{t+1}}\right)+4 n^{s}\left(\frac{2}{2^{t+1}}\right)+2 n^{s}\left(\frac{3}{2^{t+1}}\right)+n^{s}\left(\frac{4}{2^{t+1}}\right)\right] \\
& =\frac{1}{n}\left[4 n^{s}\left(\frac{2}{2^{t+1}}\right)+n^{s}\left(\frac{4}{2^{t+1}}\right)\right] \\
& =\frac{1}{n}\left[4 n^{s}\left(\frac{1}{2^{t}}\right)+n^{s}\left(\frac{2}{2^{t}}\right)\right] \\
H^{t+1}\left(\frac{3}{2^{t}} ; s\right) & =\frac{1}{n}\left[32 n^{s}\left(\frac{1}{2^{t+1}}\right)+16 n^{s}\left(\frac{2}{2^{t+1}}\right)+8 n^{s}\left(\frac{3}{2^{t+1}}\right)\right.
\end{aligned}
$$




$$
\begin{aligned}
& \left.+4 n^{s}\left(\frac{4}{2^{t+1}}\right)+2 n^{s}\left(\frac{5}{2^{t+1}}\right)+n^{s}\left(\frac{6}{2^{t+1}}\right)\right] \\
= & \frac{1}{n}\left[16 n^{s}\left(\frac{2}{2^{t+1}}\right)+4 n^{s}\left(\frac{4}{2^{t+1}}\right)+n^{s}\left(\frac{6}{2^{t+1}}\right)\right] \\
= & \frac{1}{n}\left[16 n^{s}\left(\frac{1}{2^{t}}\right)+4 n^{s}\left(\frac{2}{2^{t}}\right)+n^{s}\left(\frac{3}{2^{t}}\right)\right] .
\end{aligned}
$$

Now, applying Eq. (26) to the grid $\mathcal{C}(t)$, one has:

$$
\begin{aligned}
& H^{t}\left(\frac{1}{2^{t}} ; s\right)=\frac{1}{n} n^{s}\left(\frac{1}{2^{t}}\right) \\
& H^{t}\left(\frac{2}{2^{t}} ; s\right)=\frac{1}{n}\left[2 n^{s}\left(\frac{1}{2^{t}}\right)+n^{s}\left(\frac{2}{2^{t}}\right)\right] \\
& H^{t}\left(\frac{3}{2^{t}} ; s\right)=\frac{1}{n}\left[4 n^{s}\left(\frac{1}{2^{t}}\right)+2 n^{s}\left(\frac{2}{2^{t}}\right)+n^{s}\left(\frac{3}{2^{t}}\right)\right],
\end{aligned}
$$

so that Expression (90) for $i=1,2,3$ results from combining (92)-(94) with (95)(97).

STEP 3. In order to prove the result, consider two societies $s$ and $s^{\prime}$ and assume that society $s H^{t}$ dominates society $s^{\prime}$ so that:

$$
H^{t}\left(\frac{i}{2^{t}} ; s\right) \leq H^{t}\left(\frac{i}{2^{t}} ; s^{\prime}\right)
$$

holds for all $i \in\left\{1, \ldots,\left(2^{t}\right) k\right\}$. Taking any such $i$, one has in particular:

$$
H^{t}\left(\frac{h}{2^{t}} ; s\right) \leq H^{t}\left(\frac{h}{2^{t}} ; s^{\prime}\right)
$$

for any $h \in\{1, \ldots, i\}$. Hence, using (90):

$$
H^{t+1}\left(\frac{i}{2^{t}} ; s\right) \leq H^{t+1}\left(\frac{i}{2^{t}} ; s^{\prime}\right)
$$

for all $i \in\left\{1, \ldots,\left(2^{t}\right) k\right\}$ which implies, thanks to (87), that society $s H^{t+1}$-dominates society $s^{\prime}$.

\section{A.12 Theorem 6}

The proof that Statement (a) of the theorem implies Statement (b) is established in Proposition 8 (by using $t=0$ ). In order to prove the converse implication, consider two arbitrary societies $s$ and $s^{\prime}$ such that $s \succeq_{L} s^{\prime}$. Because of Proposition 9, we only have to show that there exists a nonnegative integer $t$ for which $s H^{t}$-dominates $s^{\prime}$ holds or, equivalently thanks to Theorem 3 , that $s$ can be obtained from $s^{\prime}$ by means of a finite 
sequence of increments and/or Hammond transfers on the grid $\mathcal{C}(t)$. Since $s \succeq_{L} s^{\prime}$, there is by Definition 4 an index $i \in\{1,2, \ldots, k\}$ such that $n_{h}^{s}=n^{s}(h)=n^{s^{\prime}}(h)=n_{h}^{s^{\prime}}$ for all $h \in\{1,2, \ldots, i-1\}$ and $n_{i}^{s}=n^{s}(i)<n^{s^{\prime}}(i)=n_{i}^{s^{\prime}}$. Given this index $i$, consider a society $s^{\prime \prime}$ such that:

$$
\begin{aligned}
n_{h}^{s^{\prime \prime}} & =n_{h}^{s}, \forall h \in\{1, \ldots, i\} ; \\
n_{i+1}^{s^{\prime \prime}} & =\sum_{h=i+1}^{k} n_{h}^{s} ; \\
n_{h}^{s^{\prime \prime}} & =0, \forall h \in\{i+2, \ldots, k\} .
\end{aligned}
$$

Notice that $\sum_{h=1}^{k} n_{h}^{s^{\prime \prime}}=n$ and that $F(i ; s) \leq F\left(i ; s^{\prime \prime}\right)$ for all $i \in\{1, \ldots, k\}$ so that, by Theorem (1), $s$ can be obtained from $s^{\prime \prime}$ by means of a finite sequence of increments. We also observe that:

$$
\begin{aligned}
& n_{h}^{s^{\prime}}=n_{h}^{s^{\prime \prime}}, \forall h \in\{1, \ldots, i-1\} \\
& n_{i}^{s^{\prime}}-n_{i}^{s^{\prime \prime}}>0 ; \quad n_{i+1}^{s^{\prime}}-n_{i+1}^{s^{\prime \prime}}<0 ; \\
& n_{h}^{s^{\prime}} \geq n_{h}^{s^{\prime \prime}}=0, \quad \forall h \in\{i+2, \ldots, k\} .
\end{aligned}
$$

Define, for any $h \in \mathcal{C}$, the number $\delta_{h}$ by:

$$
\delta_{h}=n_{h}^{s^{\prime}}-n_{h}^{s^{\prime \prime}}
$$

It is clear that $\delta_{h}$ so defined is an integer (which may be positive or negative). Since $\sum_{h=i}^{k} n_{h}^{s^{\prime}}=\sum_{h=i}^{k} n_{h}^{s^{\prime \prime}}$, one can write:

$$
\delta_{i}+\delta_{i+1}=-\sum_{h=i+2}^{k} \delta_{h} .
$$

Since, by (100), $\delta_{h} \geq 0$ for all $h \in\{i+2, \ldots, k\}$, one observes that $\delta_{i}+\delta_{i+1} \leq 0$. We consider two cases.

CASE 1: $\delta_{i}+\delta_{i+1}=0$. In that case, we conclude from (101) that $\sum_{h=i+2}^{k} \delta_{h}=0$ and, thanks to (100), that $n_{h}^{s^{\prime}}=n_{h}^{s^{\prime \prime}}$ for all $h \in\{i+2, \ldots, k\}$. Hence, one has $n_{h}^{s^{\prime}}=n_{h}^{s^{\prime \prime}}$ for all $h=\{1, \ldots, i-1\} \cap\{i+2, \ldots, k\}$ and $\delta_{i}=n_{i}^{s^{\prime}}-n_{i}^{s^{\prime \prime}}=n_{i+1}^{s^{\prime \prime}}-n_{i+1}^{s^{\prime}}>0$. Hence, $s^{\prime \prime}$ can be obtained from $s^{\prime}$ by means of $\delta_{i}$ increments from $i$ to $i+1$. We conclude that $s$ first-order dominates $s^{\prime \prime}$, but also $s^{\prime \prime}$ first-order dominates $s^{\prime}$, which implies that $s H^{t}$-dominates $s^{\prime}$ for all $t \in\{0,1, \ldots\}$.

CASE 2: $\delta_{i}+\delta_{i+1}<0$. In that case, we deduce from (101) that there is an $h \in$ $\{i+2, \ldots, k\}$ such that $\delta_{h}>0$ or, equivalently, that $n_{h}^{s^{\prime}}>n_{h}^{s^{\prime \prime}}=0$. From (98)(100), one immediately observes that $s^{\prime \prime}$ can be obtained from $s^{\prime}$ by means of $\delta_{i}$ increments from category $i$ to category $i+1$, and $\left(-\delta_{i+1}\right)$ decrements $\left(\delta_{i+1}\right.$ is a negative integer), from each category $h>i+1$ for which $n_{h}^{s^{\prime}}>0$ to category $i+1$. However, more decrements than increments are required $\left(\left(-\delta_{i+1}\right)>\delta_{i}\right.$, so 
that increments and decrements cannot be matched one by one to produce Hammond transfers - and only Hammond transfers - in order to obtain, on the initial grid $\mathcal{C}$, $s^{\prime \prime}$ from $s^{\prime}$. Yet, we can match the increments with the decrements if an appropriate refinement of the grid between $i$ and $i+1$ can be performed. First, staying on the initial grid $\mathcal{C}$, and starting from $s^{\prime}$, we can combine $\left(\delta_{i}-1\right)$ increments (from $i$ to $i+1$ ) to the same number of decrements starting from one or several categories $h$ above $i+1$ and bringing the agents from these categories to $i+1$. This generates immediately $\left(\delta_{i}-1\right)$ Hammond transfers. In order to complete the move from $s^{\prime}$ to $s$ by means of Hammond transfers, we need to match the last $\left[\delta_{i}-\left(\delta_{i}-1\right)\right]=1$ increment from $i$ to $i+1$ with the remaining $\left[\left(-\delta_{i+1}\right)-\left(\delta_{i}-1\right)\right]>1$ decrements that are required from each category $h>i+1$ where the number of agents remains strictly positive to the category $i+1$. Whatever is the number $\left[\left(-\delta_{i+1}\right)-\left(\delta_{i}-1\right)\right]>1$, it is clearly possible to refine the grid $\mathcal{C}$ in such a way as to obtain at least $\left[\left(-\delta_{i+1}\right)-\left(\delta_{i}-1\right)\right]$ adjacent categories between $i$ and $i+1$. Once this refinement is obtained, one can then proceed in decomposing the last increment from $i$ to $i+1$ into $\left[\left(-\delta_{i+1}\right)-\left(\delta_{i}-1\right)\right]$ "small" increments between adjacent intermediate categories, each of which being matched with a decrement from each category $h>i+1$ for which there is a strictly positive number of agents. Hence, it is possible to achieve $s^{\prime \prime}$ from $s$ by using Hammond transfers only (provided that a suitable refinement of the grid be performed). Hence, there exists a nonnegative integer $t$ such that $s^{\prime \prime}$ can be obtained from $s^{\prime}$ by means of exactly $\left(-\delta_{i+1}\right)$ Hammond transfers on the grid $\mathcal{C}(t)$ (recalling that a transformation on the grid $\mathcal{C}$ is also a transformation on the grid $\mathcal{C}(t)$ ). We then conclude that society $s$ first-order dominates society $s^{\prime \prime}$ which in turn $H^{t}$-dominates society $s^{\prime}$ and this completes the proof.

\section{References}

Abul-Naga, R.: Measurement of inequality with finite number of pay states: the majorization set and its applications. Econ. Theory 65, 99-123 (2018). https://doi.org/10.1007/s00199-016-1011-2

Abul-Naga, R., Yalcin, T.: Inequality measurement for ordered response health data. J. Health Econ. 27, 1614-1625 (2008)

Allison, R.A., Foster, J.E.: Measuring health inequality using qualitative data. J. Health Econ. 23, 505-524 (2004)

Apouey, B.: Measuring health polarization with self-assessed health data. Health Econ. 16, 875-894 (2007)

Atkinson, A.B.: On the measurement of inequality. J. Econ. Theory 2, 244-263 (1970)

Blackorby, C., Bossert, W., Donaldson, D.: Population Issues in Social Choice Theory. Cambridge University Press, Cambridge (2005)

Bosmans, K., Ooghe, E.: A characterization of maximin. Econ. Theory Bull. 1, 151-156 (2013)

Bosmans, K., Lauwers, L., Ooghe, E.: Prioritarian poverty comparisons with cardinal and ordinal attributes. Scand. J. Econ. 120, 925-942 (2018)

Castelló-Clement, A., Doménech, R.: Human capital inequality and economic growth: some new evidence. Econ. J. 112, C187-C200 (2002)

Castelló-Clement, A., Doménech, R.: Human capital inequality, life expectancy and economic growth. Econ. J. 118, 653-677 (2008)

Chakravarty, S., Maharaj, B.: Generalized Gini polarization indices for an ordinal dimension of human well-being. Int. J. Econ. Theory 11, 231-246 (2015)

Chakravarty, S., Zoli, C.: Stochastic dominance relations for integer variables. J. Econ. Theory 147, 13311341 (2012)

Cowell, F.A., Flachaire, E.: Inequality with ordinal data. Economica 84, 290-321 (2017) 
Dalton, H.: The measurement of the inequality of incomes. Econ. J. 30, 348-361 (1920)

Dasgupta, P., Sen, A.K., Starrett, D.: Notes on the measurement of inequality. J. Econ. Theory 6, 180-187 (1973)

D’Aspremont, C.: Axioms for social welfare orderings. In: Hurwicz, L., Schmeidler, D., Sonnenschein, H. (eds.) Social Goals and Social Organization, pp. 19-76. Cambridge University Press, Cambridge (1985)

D’Aspremont, C., Gevers, L.: Equity and the informational basis of social choice. Rev. Econ. Stud. 46, 199-210 (1977)

Deschamps, R., Gevers, L.: Leximin and utilitarian rules: a joint characterization. J. Econ. Theory 17, 143-163 (1978)

Fields, G., Fei, J.: On inequality comparisons. Econometrica 46, 305-316 (1978)

Fishburn, P.C., Lavalle, I.H.: Stochastic dominance on unidimensional grids. Math. Oper. Res. 20, 513-525 (1995)

Fleurbaey, M., Maniquet, F.: Well-being measurement with non-classical goods. Econ. Theory 68, 765-786 (2019). https://doi.org/10.1007/s00199-018-1143-7

Gravel, N., Marchant, T., Sen, A.: Comparing societies with different numbers of individuals on the basis of their average advantage. In: Fleurbaey, M., Salles, M., Weymark, J.A. (eds.) Social Ethics and Normative Economics: Essays in Honour of Serge-Christophe Kolm, pp. 261-277. Springer, Berlin (2011)

Gravel, N., Magdalou, B., Moyes, P.: Inequality measurement with an ordinal and continuous variable. Soc. Choice Welf. 52, 453-475 (2019)

Hammond, P.J.: Equity, Arrow's conditions and Rawls's difference principle. Econometrica 44, 793-803 (1976)

Hammond, P.J.: Equity in two person situations: some consequence. Econometrica 47, 1127-1135 (1979)

Hardy, G.H., Littlewood, J.E., Polya, G.: Inequalities, 2nd edn. Cambridge University Press, Cambridge (1952)

Kobus, M.: Polarization measurement for ordinal data. J. Econ. Inequal. 13, 275-277 (2015)

Kobus, M., Milós, P.: Inequality decomposition by population subgroups for ordinal data. J. Health Econ. 31, 15-21 (2012)

Kolm, S.C.: The optimal production of social justice. In: Guitton, H., Margolis, J. (eds.) Public Economics. Macmillan, London (1969)

Lehmann, E.L.: Ordered family of distributions. Ann. Math. Stat. 26, 399-419 (1955)

Magdalou, B.: An model of social welfare improving transfers. Center for Environmental Economics Montpellier, Working paper no. 2018-13 (2018)

Marshall, A.W., Olkin, I., Arnold, B.C.: Inequalities: Theory of Majorization and Its Applications, 2nd edn. Springer, New York (2011)

Miyagishima, K.: A characterization of the maximin social ordering. Econ. Bull. 30, 1278-1282 (2010)

Muller, A., Scarsini, M.: Fear of loss, inframodularity and transfers. J. Econ. Theory 147, 1490-1500 (2012)

Pradhan, M., Sahn, D.A., Younger, S.D.: Decomposing world health inequality. J. Health Econ. 22, 271-293 (2003)

Quirk, J.D., Saposnik, R.: Admissibility and measurable utility functions. Rev. Econ. Stud. 29, 140-146 (1962)

Sen, A.K.: On Economic Inequality. Clarendon, Oxford (1973)

Sen, A.K.: On weights and measures: informational constraints in social welfare analysis. Econometrica 45, 1539-1572 (1977)

Shorrocks, A.F.: Ranking income distributions. Economica 50, 3-17 (1983)

Tungodden, B.: Egalitarianism: Is Leximin the only option? Econ. Philos. 16, 229-245 (2000)

Zheng, B.: A note on measuring inequality with ordinal data. In: Bishop, J., Zheng, B. (eds.) Research in Economic Inequalities, vol. 16, pp. 177-188. Bingley, Emerald (2008)

Zheng, B.: A new approach to measure socioeconomic inequality in health. J. Econ. Inequal. 9, 555-577 (2011) 


\section{Affiliations}

\section{Nicolas Gravel ${ }^{1}\left[\right.$. Brice Magdalou ${ }^{2} \cdot$ Patrick Moyes $^{3}$}

Brice Magdalou

brice.magdalou@umontpellier.fr

Patrick Moyes

patrick.moyes@u-bordeaux.fr

1 Centre de Sciences Humaines \& Aix-Marseille Université, CNRS, EHESS, ECM, AMSE, 2, Dr. A.P.J. Abdul Kalam Road, Delhi 110011, India

2 CEE-M. Univ. Montpellier, CNRS, INRAE, SupAgro, Montpellier, France

3 GREThA, CNRS, UMR 5113, Université de Bordeaux, Avenue Léon Duguit, 33608 Pessac, France 